\title{
A metabolic and genomic study of engineered Saccharomyces cerevisiae strains for high glycerol production
}

\author{
Hélène Cordier ${ }^{\mathrm{a}, 1,2}$, Filipa Mendes ${ }^{\mathrm{b}, 1}$, Isabel Vasconcelos ${ }^{\mathrm{b}}$, Jean M. François ${ }^{\mathrm{a}, *}$ \\ ${ }^{a}$ Laboratoire de Biotechnologie et Bioprocédés, UMR-CNRS 5504 \& INRA 792, Toulouse, France \\ ${ }^{\mathrm{b}}$ Escola Superior de Biotecnologia, Universidade Católica Portuguesa, Porto, Portugal
}

Keywords: Glycerol metabolism; Genetic engineering; Metabolic regulation; Transcriptomic analysis; Saccharomyces cerevisiae

\begin{abstract}
Towards a global objective to produce chemical derivatives by microbial processes, this work dealt with a metabolic engineering of the yeast Saccharomyces cerevisiae for glycerol production. To accomplish this goal, overexpression of GPD1 was introduced in a tpil $\triangle$ mutant defective in triose phosphate isomerase. This strategy alleviated the inositol-less phenotype of this mutant, by reducing the levels of dihydroxyacetone phosphate and glycerol-3-P, two potent inhibitors of myo-inositol synthase that catalyzes the formation of inositol6-phosphate from glucose-6-phosphate. Further deletion of $A D H 1$ and overexpression of $A L D 3$, encoding, respectively, the major $\mathrm{NAD}^{+}$-dependent alcohol dehydrogenase and a cytosolic $\mathrm{NAD}^{+}$-dependent aldehyde dehydrogenase yielded a yeast strain able to produce $0.46 \mathrm{~g}$ glycerol (g glucose $)^{-1}$ at a maximal rate of $3.1 \mathrm{mmol}(\mathrm{g} \text { dry mass })^{-1} \mathrm{~h}^{-1}$ in aerated batch cultures. At the metabolic level, this genetic strategy shifted the flux control coefficient of the pathway to the level of the glycerol efflux, with a consequent intracellular accumulation of glycerol that could be partially reduced by the overproduction of glycerol exporter encoded by FPS1. At the transcriptomic level, this metabolic reprogramming brought about the upregulation of genes encoding $\mathrm{NAD}^{+} / \mathrm{NADP}^{+}$binding proteins, a partial derepression of genes coding for TCA cycle and respiratory enzymes, and a downregulation of genes implicated in protein biosynthesis and ribosome biogenesis. Altogether, these metabolic and molecular alterations stand for major hurdles that may represent potential targets for further optimizing glycerol production in yeast.
\end{abstract}

\section{Introduction}

Glycerol is one of the most important by-products of glucose conversion during alcoholic fermentation by Saccharomyces cerevisiae. This metabolite can reach $0.1 \mathrm{~g}$ per g glucose consumed under anaerobic condition to equilibrate the redox balance by regenerating $\mathrm{NADH}$ associated with the biomass production during the fermentation (Overkamp et al., 2002; Remize et al., 1999). Glycerol is also involved in osmoregulation (for a review see (Hohmann, 2002), and can act as a cryoprotec-

\footnotetext{
*Corresponding author. Laboratoire de Biotechnologie et Bioprocédés, Institut National des Sciences Appliquées, Avenue de Rangeuil, F-31077 Toulouse, Cedex 04, France.

E-mail address: fran_jm@insa-toulouse.fr (J.M. François).

${ }^{1}$ Both authors have equally contributed to this work.

${ }^{2}$ Present address: SAF-ISIS, ZA, 40140 Soustons, France.
}

tant like trehalose (Izawa et al., 2004). As shown in Fig. 1, glycerol is synthesized in the yeast $S$. cerevisiae in a two reaction process, consisting of the reduction of dihydroxyacetone phosphate (DHAP) by a $\mathrm{NAD}^{+}$-dependent glycerol-3-phosphate dehydrogenase (GPD), followed by the dephosphorylation of glycerol-3-phosphate (glycerol-3P) by a glycerol-3-phosphate phosphatase (GPP) (Albertyn et al., 1994b; Ansell et al., 1997; Pahlman et al., 2001). GPD1 and GPD2 encode the two isozymes of GPD (Larsson et al., 1993) and GPP1 and GPP2, those of GPP (Pahlman et al., 2001). These genes are subject to different control, e.g., expression of GPD1 and GPP2 are increased in response to an osmotic stress, while GPD2 and GPP1 are induced under anaerobic condition (Albertyn et al., 1994b; Ansell et al., 1997; Costenoble et al., 2000; Pahlman et al., 2001). Based on these considerations and on other genetic data, it is assumed that the couple Gpd1p/Gpp2p is 


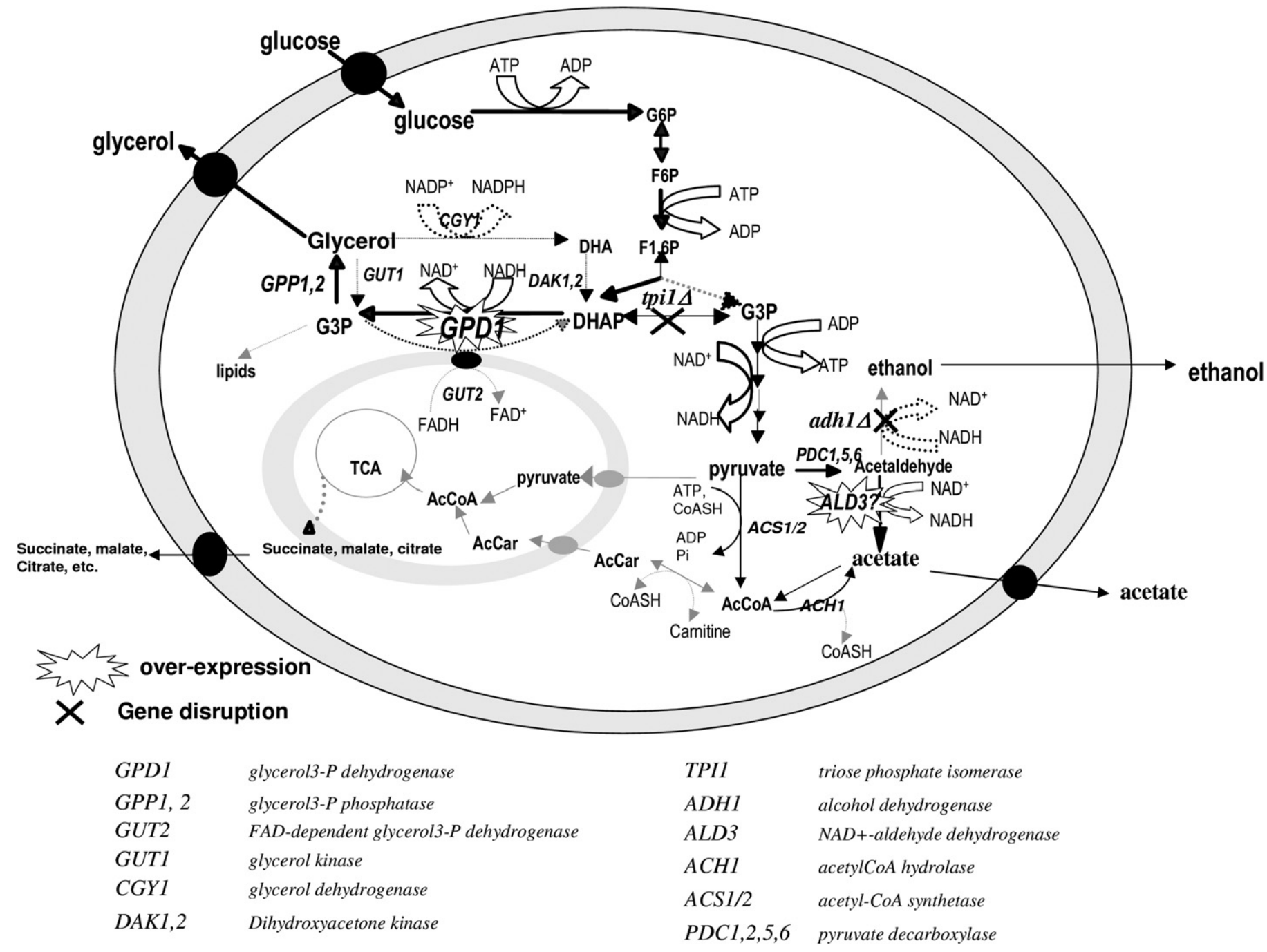

Fig. 1. Strategy of genetic engineering of Saccharomyces cerevisiae for high glycerol production.

the major route for glycerol production under aerobic condition, whereas Gpd2p/Gpplp play a specific role in redox balance under anaerobic growth conditions (Blomberg, 1997; Hohmann, 2002). Additionally, the distinct localization of Gpd1p and Gpd2p may also explain that the former cannot substitute for the latter in mitochondrial defective strains (Valadi et al., 2004).

The yeast $S$. cerevisiae is not only a good glycerol producer, it is also the most useful system to evaluate various engineering strategies to optimize the synthesis of this product. Over the past decade, there have been several attempts at manipulating yeast metabolism to optimize glycerol production by shifting from a bioprocess-mediated mode involving sulphite to a reprogramming of the cellular metabolism by recombinant DNA technology. The latter strategy has been relatively successful since it raised the glycerol production from $0.25 \mathrm{~g}$ glycerol $(\mathrm{g} \text { glucose })^{-1}$ obtained by the sulphite bioprocess (Bisping and Rehm, 1988; Petrovska et al., 1999) to $0.42 \mathrm{~g}$ glycerol (g glucose) ${ }^{-1}$ by a metabolic engineering strategy that involved a deletion of TPII encoding triose phosphate isomerase and the removal of the cytosolic NADH reoxidation by mitochondrial redox shuttles (Overkamp et al., 2002). In a recent paper, Pronk and coworkers could reach a glycerol yield close to $0.50 \mathrm{~g}$ glycerol (g glucose) $^{-1}$ by another genetic engineering strategy that overcame TPII deletion, but that required a disruption of genes encoding respiratory chainlinked NADH dehydrogenase and pyruvate decarboxylase (Geertman et al., 2006).

In spite of the relative technological advantages of using $S$. cerevisiae to put into practice various genetic strategies, the remaining challenge is to get the maximal glycerol yield at the fastest rate. This achievement requires a reduction of the contribution of assimilatory glucose metabolism to anabolic and maintenance purposes and an increase in the NADH availability. These two constraints could eventually be overcome by a comprehensive investigation of the consequences of the genetic engineering strategies at the metabolic and genomic levels. As depicted in Fig. 1, our strategy comprised the over-expression of GPD1 encoding glycerol phosphate dehydrogenase, followed by a disruption of TPII and $A D H 1$, which encode, respectively, the 
triose phosphate isomerase and the major alcohol dehydrogenase. In addition, we evaluated whether the overexpression of a $\mathrm{NAD}^{+}$-dependent aldehyde dehydrogenase encoded by $A L D 3$ (Navarro-Avino et al., 1999) could reduce the increased levels of acetaldehyde generated by these genetic modifications in favour of a supplementary provision of NADH for glycerol production. Overall, this strategy yielded an engineered strain that was able to produce $0.46 \mathrm{~g}$ glycerol (g glucose $)^{-1}$ at a production rate of $3.1 \mathrm{mmol}(\mathrm{g} \text { biomass } \mathrm{h})^{-1}$. Our metabolic and transcriptomic analyses of the engineered strains highlighted many cellular targets that can be responsible for restraining yeast cells for higher glycerol productivity.

\section{Materials and methods}

\section{Construction of engineered yeast strains}

All $S$. cerevisiae strains constructed in this study are listed in Table 1. The host strain was the wild type auxotrophic strain of the diploid CEN.PK2 family described in van Dijken et al. (2000). Gene were integrated/deleted into/from yeast genome by homologous recombination following the transformation procedure of Woods and Gietz (2001). Verification of genetic modification was carried out by diagnostic PCR using specific primers listed in Table 2. Selective media for yeast transformants and genetic manipulation of yeast cells for sporulation and tetrad dissection were carried out as described (Rose et al., 1990).

For overexpression of GPDI in CEN.PK2, the ORF (1176 bp) was amplified by PCR from genomic DNA using

Table 1

Strains used in this work

\begin{tabular}{|c|c|c|}
\hline Strain & Genotype & References \\
\hline CENPK2 & 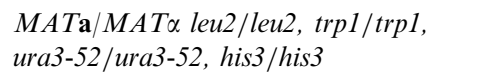 & $\begin{array}{l}\text { van Dijken et al. } \\
(2000)\end{array}$ \\
\hline $\mathrm{HC} 13$ & $\begin{array}{l}\text { MAT } \alpha \text { leu2::GPD1-LEU2, trp1, ura3- } \\
52, \text { his3 }\end{array}$ & This work \\
\hline HC14 & 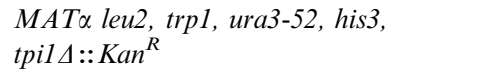 & This work \\
\hline $\mathrm{HC} 16$ & $\begin{array}{l}\text { MAT } \alpha \text { leu } 2:: G P D 1-L E U 2, \text { trp } 1, \text { ura3- } \\
52, \text { his } 3, \text { tpil ::Kan }\end{array}$ & This work \\
\hline $\mathrm{HC} 17$ & $\begin{array}{l}\text { MAT } \alpha \text { adh1 ::Kan }{ }^{R} \text { leu } 2 \text { trp1 ura3-52 } \\
\text { his } 3\end{array}$ & This work \\
\hline $\mathrm{HC} 23$ & $\begin{array}{l}\text { MAT } \alpha \text { leu2 }:: \text { GPD1-LEU2 } \\
\operatorname{trp} 1:: \text { ALD3-TRP1ura3-52 his3 }\end{array}$ & This work \\
\hline HC30 & 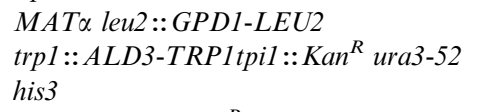 & This work \\
\hline HC32 & $\begin{array}{l}\text { MATa adh1 }:: \text { Kan }^{R} \text { leu } 2:: \text { GPD1-LEU2 } \\
\text { tpil :: Kan }{ }^{R} \text { trp1 ura3-52 his } 3\end{array}$ & This work \\
\hline $\mathrm{HC} 42$ & $\begin{array}{l}\text { MATa adh1::Kan }{ }^{r} \text { leu2 }:: \text { GPD1-LEU2 } \\
\text { tpil:: Kan }{ }^{r} \text { trp1::ALD3-TRP1 ura3-52 } \\
\text { his3 }\end{array}$ & This work \\
\hline FM62 & $\begin{array}{l}\text { MATa adh1 :: Kan }{ }^{r}, \text { leu2 }:: G P D 1-L E U 2, \\
\text { tpil::Kan }{ }^{r} \text {, trp } 1:: A L D 3-T R P 1, \text { ura3- } \\
52, \text { his3, ADH1-FPS1::URA3 }\end{array}$ & This work \\
\hline
\end{tabular}

primers $\mathrm{HCl}$ and $\mathrm{HC} 2$, flanked with HindIII linkers (restriction site underlined in Table 2). The PCR fragment was cloned into the pGEMT (purchased from Promega) to yield $\mathrm{pHCl}$. $A D H 1$ promoter and terminator were amplified by PCR using the plasmid pODB80 (Louvet et al., 1997) as DNA template with primers $\mathrm{HC} 3$ and HC4 for $A D H 1$ promoter, and HC5 and HC6 for $A D H 1$ terminator, respectively (Table 2). The primers were designed to create a HindIII cloning site between $A D H 1$ promoter and terminator. This amplification generated two fragments (705 pb and $193 \mathrm{bp}$ ) that were cloned together in the $\mathrm{XhoI} /$ SpeI sites of pRS305 to generate pHC12. Then, the $1176 \mathrm{bp}$ HindIII fragment of $\mathrm{pHCl}$ was cloned into the HindIII cloning site of $\mathrm{pHC} 12$ to yield $\mathrm{pHC} 13$ (GPDI ORF under the $A D H 1$ promoter). The diploid strain CEN.PK2 was transformed with the linearized $\mathrm{pHC13}$ plasmid (cut by EcoRI in the LEU2 marker) and transformants were selected on SD leu ${ }^{-}$selective medium to retain one clone of each opposite mating type ( $\mathrm{HC13}$ a and $\mathrm{HCl} 3 \alpha)$ that was verified by PCR for correct integration of GPDI at the Leu locus. The two clones were crossed to get $\mathrm{HC} 13 \mathbf{a} / \alpha$ diploid cells.

Mutant strain deleted for TPII and overexpressing GPDI was constructed as follows. TPII encoding triose isomerase was disrupted using the loxP-KanMX4-loxP cassette (Guldener et al., 1996) that was PCR-amplified with $40 \mathrm{bp}$ homologous to yeast TPII gene flanked upstream and downstream of the cassette using primers HC9 and HC10 (Table 2). The purified cassette was used to transform the diploid $\mathrm{HC13} \mathbf{a} / \alpha$ strain. Transformants were selected on YPD medium containing $0.2 \mathrm{gl}^{-1}$ G418 according to Wach et al. (1994). Heterozygote clones identified by PCR for correct disruption of TPII was then sporulated and dissected on MO (1\% yeast extract, $2 \%$ bactopeptone, $0.1 \%$ glucose and $2 \%$ ethanol) agar plate and then replica plated on YPD $+0.2 \mathrm{gl}^{-1} \mathrm{G} 418$, SD supplemented with leucine, uracil, tryptophane and histidine, and SD supplemented with same auxotrophic requirements except leucine, both media also containing $100 \mu \mathrm{M}$ myo-inositol to select for tpil 4 and tpil $A$ GPIoverexpressing clones.

Deletion of $A D H 1$ was also obtained by the short flanking homology method using pUG6 as the template (Guldener et al., 1996). The cassette was used to transform CEN.PK2 diploid strain, and the haploid strain $\mathrm{HC} 17$ was isolated after sporulation of one $A D H 1 / a d h 1 \Delta$ heterozygous diploid. Overexpression of $A L D 3$ encoding a cytosolic $\mathrm{NAD}^{+}$-dependent aldehyde dehydrogenase (Navarro-Avino et al., 1999; White et al., 2003) was carried out by PCR amplification of the ALD3 ORF using genomic DNA in two step procedure, because of the high identity shared with $A L D 2(98 \%)$. In the first step, the gene (from -73 to +1684 ) was amplified using primers $\mathrm{HC} 13$ and $\mathrm{HC} 14$ (Table 2) and subcloned into pGEMT. In a second step, the $A L D 3$ ORF (from ATG to stop codon) was amplified with $\mathrm{HC} 15$ and $\mathrm{HC} 16$ (each primer contained a Pst $\mathrm{I}$ linker at their $5^{\prime}$ end) and recloned into pGEMT to 
Table 2

Oligonucleotides sequences for the construction of gene overexpression or deletion cassettes and for diagnostic PCR of the engineered strains

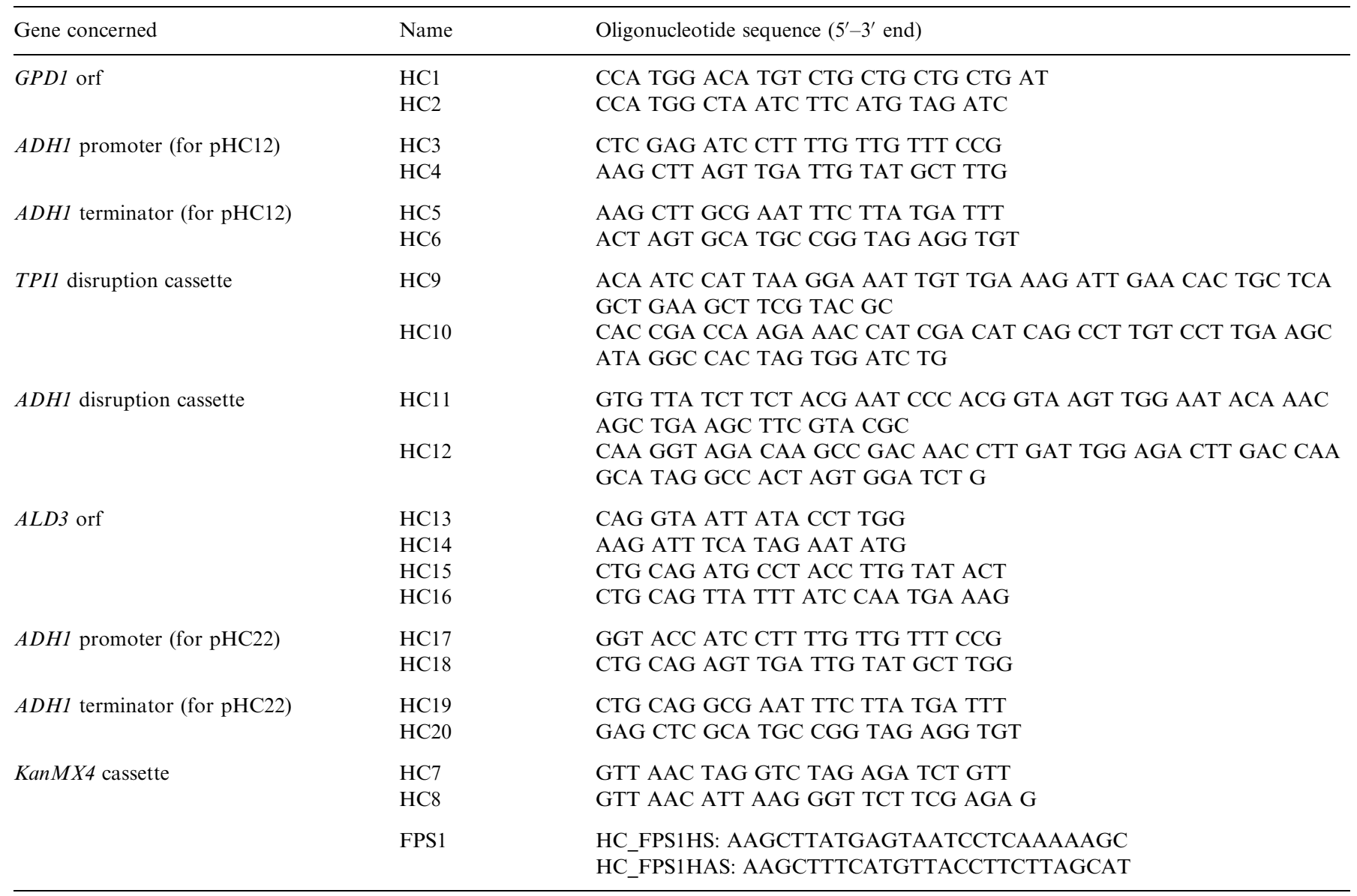

yield pHC24. To express $A L D 3$ under $A D H 1$ promoter, we modified pRS304 as follows. The $A D H 1$ promoter and terminator were amplified by PCR from pODB80 using primers $\mathrm{HC} 17$ and $\mathrm{HC} 18$ (for $A D H 1$ promoter) and $\mathrm{HC} 19$ / $\mathrm{HC} 20$ (for $A D H 1$ terminator). These primers bear at their $5^{\prime}$ end a PstI cloning site and they were cloned into KpnI and $\mathrm{SacI}$ sites of the integrative plasmid pRS304 (Sikorski and Hieter, 1989). Then, ALD3 ORF from pHC24 was digested by PstI and cloned into the modified pRS304 to yield $\mathrm{pHC} 23$. This plasmid was linearized by cutting in the $T R P 1$ marker with $X b a$ I to target homologous recombination at TRP1 locus into HC23 strain. Transformants were selected on SD $\operatorname{trp}^{-}$medium.

Haploid strain HC30 (leu2 :: GPD1-LEU2 tpil ::KanMX trp1::ALD3-TRP1 trp1 ura3 his3) was obtained from diploid $\mathrm{HC} 16 \times \mathrm{HC} 30 \quad(M A T \alpha \quad l e u 2:: G P D 1-L E U 2$ tpil::KanMX4 trp1 ura3 his3) and HC23 (MATa leu2::GPD1-LEU2 trp1::ALD3-TRP1, trp1 ura3 his3) by tetrad analysis. The presence of $G P D 1$ and $A L D 3$ cassettes and tpil $\triangle$ disruption was checked by PCR. This allowed us to isolate the haploid strain HC30 (leu2::GPD1-LEU2 trp1::ALD3-TRP1 tpil ::KanMX4 ura3 his3). Strain HC32 (adh1 ::KanMX4 leu2 :: GPD1-LEU2, tpil ::KanMX4 trp1 ura3 his3) was obtained by crossing HC16 (MATa leu2::GPD1-LEU2 tpil::KanMX4 trp1 ura3 his3) with
HC17 (MATa adh1::KanMX4 leu2, trp1 ura3 his3). This haploid strain $\mathrm{HC} 32$ was also transformed with the linearized integrative $\mathrm{pHC} 23$ plasmid to integrate $A L D 3$ at the TRPI locus by homologous recombination to yield HC42. For overexpression of FPS1 in HC42, the ORF was amplified by PCR from genomic DNA using to primers HC_FPS1HS and HC_FPS1HAS, flanked with HindIII linkers. The PCR fragment was cloned in pGEMT (digestion HindIII). The generated fragment was subcloned into the HindIII cloning site of pHC 32 (URA3 marker) under $A D H 1$ promoter, to yield pHC33. This plasmid was linearized by cutting the URA3 marker with $E c o R V$ in order to introduce the construct by homologous recombination at the URA3 locus into $\mathrm{HC} 42$ strain. Transformants were selected on SD $\mathrm{ura}^{-}$medium, and selected clones were verified by PCR for correct integration.

\section{Shake fask and batch cultures}

To assess for growth efficiency, the wild type and engineered yeast strains were cultivated at $30^{\circ} \mathrm{C}$ in 0.51 Erlenmeyer flasks containing $150 \mathrm{ml}$ of the following media: YPD ( $2 \%$ peptone, $1 \%$ yeast extract, $2 \%$ glucose), MO $(2 \%$ peptone, $1 \%$ yeast extract, $0.1 \%$ glucose, $1 \%$ 
ethanol), SD ( $1.7 \mathrm{~g}^{-1}$ yeast nitrogen base without amino acids and ammonium, $5 \mathrm{~g} \mathrm{l}^{-1}$ ammonium sulphate, $20 \mathrm{gl}^{-1}$ glucose and completed with auxotrophic requirements) buffered at $\mathrm{pH} 5.0$ by addition of $85 \mathrm{mM}$ sodium succinate $\mathrm{pH}$ 5.0. Growth was followed by measurement of absorbance at $600 \mathrm{~nm}$.

For cultivation in bioreactors, a loopful of the stock culture was inoculated into two 0.51 Erlenmeyer flasks containing $150 \mathrm{ml}$ of SD medium supplemented with auxotrophic requirements at $200 \mu \mathrm{g} \mathrm{ml}^{-1}$, and incubated on a rotary shaker at $150 \mathrm{rpm}$. After $24 \mathrm{~h}$ of cultivation at $30{ }^{\circ} \mathrm{C}$, the volume necessary to start the fermentation with $0.05 \mathrm{OD}$ was harvested by centrifugation and the pellet was transferred to a 2-1 bioreactor (Biostat MD, Braun, Melsungen, Germany) containing 1.51 of the same medium as in shake flasks. Cultures were carried out at $30^{\circ} \mathrm{C}$, with continuous stirring at $150 \mathrm{rpm}$ and an aeration rate of $0.5 \mathrm{vvm}$. The $\mathrm{pH}$ was maintained at 5.0 by automatic addition of $\mathrm{NaOH} 1 \mathrm{M}$.

\section{DNA microarrays analysis}

The wild type strain and engineered $\mathrm{HC} 42$ strain were harvested in early log phase $\left(\mathrm{OD}_{600}=1.0\right.$ unit $)$ by centrifugation from four independent cultures made in $\mathrm{SD}$. The frozen cells (equivalent to 10 unit $\mathrm{OD}_{600}$ ) were mechanically disrupted (MicroDismembrator Braun, Melsungen) and total RNA was isolated using RNeasy Mini kit (Qiagen) following the protocol of the manufacturer. The quantity and the quality of the extracted RNA were determined by microcapillary electrophoresis using a Bioanalyzer 2100 (Agilent Technologies, Wilmington, DE, USA). Incorporation of Cyanine 3- and Cyanine 5dCTP (Amersham Bioscience) was performed during reverse transcription of total RNA using LabelStar Reverse Transcriptase (Qiagen). Labelled cDNA was purified on MinElute spin columns (Qiagen) and was hybridized on dendrimer-activated glass slides, which bears the whole yeast genome by covalently attached DNA probes (70-mer oligonucleotides) made according to LeBerre et al. (2003). Hybridization was carried out in an automatic hybridization chamber (Discovery from Ventana Medical System, Inc) for $10 \mathrm{~h}$ at $42{ }^{\circ} \mathrm{C}$. After hybridization, the slides were washed in $2 \times \mathrm{SSC} / 0.2 \%(\mathrm{v} / \mathrm{v}) \mathrm{SDS}$, immersed briefly in isopropanol and then dried under a stream of air. Biological replicates of DNA arrays experiments were made by using total RNA that were extracted from four independent cultures (four cultures for both wild type and the engineered strains). Transcripts from two independent cultures (for both wild type and engineered strains) were retro transcribed using dCTPCY3, and the two other reciprocally with dCTP-CY5. The labelled cDNA from wild type and engineered strain were competitively hybridized on DNA microarrays. This resulted in four DNA arrays, with eight intensity values for each gene, since each gene was represented 2 times on an array. The hybridization signal was detected by scanning using GenePix 4000B laser Scanner (Axon Instruments), and the signal quantification was transformed to numerical values using the integrated GenePix software version 3.01.

The data were statistically treated using Bioplot/Bioclust software accessible at http://biopuce.insa-toulouse.fr/ ExperimentExplorer/doc/BioPLot/. Briefly, raw intensities were corrected for the local background, log transformed and normalized by the mean log-intensity of all spots. Logratios of normalized intensities from quadruplicate samples were tested for statistical significance using Student's $t$-test with Benjamini and Hockberg test correction (Hochberg and Benjamini, 1990) with a prediction for false discovery rate to about $5 \%$ of the genes identified. The differentially expressed genes were further narrowed with fold changes in expression of at least 1.5 -fold. They were classified according to functional categories following MIPS (http://mips.gsf.de/ proj/funcatDB/search_main_frame.html). Other publicly available Web resources for data expression analysis were used including Go Term Finder (http://yeastgenome.org) and FunSpec (http://funspec.med.utoronto.ca/) for Gene ontology classification and YEASTract (http://www. yeastract.com/) to search for transcriptional factors.

\section{Enzymatic assays}

Crude extracts were prepared by vortexing at $4{ }^{\circ} \mathrm{C} 4$ times $30 \mathrm{~s}$, about $25 \mathrm{mg}$ (dry mass) yeast cells with $1 \mathrm{~g}$ glass beads $(0.4-0.5 \mathrm{~mm}$ diameter) with $0.5 \mathrm{ml}$ of $20 \mathrm{mM}$ Hepes pH 7.1, $20 \mathrm{mM} \mathrm{KCl}, 1 \mathrm{mM}$ EDTA, $1 \mathrm{mM}$ DTT and protease inhibitor cocktail from Boehringer (1 capsule for $10 \mathrm{ml}$ of buffer). After centrifugation at $8000 \mathrm{~g}$ for $15 \mathrm{~min}$ at $4{ }^{\circ} \mathrm{C}$, the supernatants were used for enzymatic assays. Glycerol-3-phosphate dehydrogenase and glycerol-3-phosphate phosphatase activities were determined as described by Gancedo et al. (1968), except that the reaction buffer was $20 \mathrm{mM}$ Hepes, $\mathrm{pH} 7.1$, and the final concentration of DHAP and glycerol-3-P were 5 and $10 \mathrm{mM}$, respectively. The $\mathrm{NAD}^{+}$and $\mathrm{NADP}^{+}$-dependent glycerol-dehydrogenase were measured according to Vries et al. (2003) at $\mathrm{pH}$ 7.1 or $\mathrm{pH}$ 9.0. $\mathrm{NAD}^{+}$and $\mathrm{NADP}^{+}$-dependent acetaldehyde dehydrogenase activities were measured according to Postma et al. (1989). Alcohol dehydrogenase activity was determined in $50 \mathrm{mM} \mathrm{KH}_{2} \mathrm{PO}_{4}, \mathrm{pH} 8.0$ in the presence of $1.0 \mathrm{mM} \mathrm{NAD}{ }^{+}$and the reaction was started by the addition of $100 \mathrm{mM}$ ethanol. Glucose-6-phosphate dehydrogenase (G6PD) and glucose-6-phosphate isomerase (PGI) were measured in $20 \mathrm{mM}$ Hepes, $\mathrm{pH} 7.1$ in the presence of $0.4 \mathrm{mM} \mathrm{NADP}^{+}$. For G6PD, the reaction was started with $4.5 \mathrm{mM}$ glucose-6-phosphate. For PGI activity, the reaction mixture also contained $1 \mathrm{U} \mathrm{ml}^{-1}$ glucose-6phosphate-dehydrogenase (Sigma) and was started by addition of $1.5 \mathrm{mM}$ fructose- 6 -phosphate. The enzymatic assays were performed at $30^{\circ} \mathrm{C}$. Protein concentration was determined with the Bradford method (Bio-Rad Kit assay $\mathrm{n}^{\circ}$ 500-002) using bovine serum albumin as a standard. 


\section{Analytical procedures}

Glucose, glycerol, ethanol, acetate, acetoin and acetaldehyde were determined in the growth medium with commercial biochemical kits or by high performance liquid chromatography. In the latter case, the supernatant was filtered through $0.22 \mu \mathrm{m}$-pore-size nylon filters prior to loading on a HPX-87H Aminex ion exclusion column. The column was eluted at $30^{\circ} \mathrm{C}$ with $1 \mathrm{mM} \mathrm{H}_{2} \mathrm{SO}_{4}$ at a flow rate of $0.5 \mathrm{ml} \mathrm{min} \mathrm{m}^{-1}$ and the concentration of the compounds was determined using a Waters model 410 refractive index detector. Intracellular metabolites were extracted from whole yeast cells as described in Gonzalez et al. (1997), except for DHAP and glycerol-3-phosphate, which were extracted in $10 \% \mathrm{HClO}_{4}$ according to François et al. (1984). These two latter metabolites as well as $\mathrm{NAD}^{+}$and NADH were measured by fluorescence spectrophotometry (excitation wavelength $340 \mathrm{~nm}$, emission wavelength $460 \mathrm{~nm}$ ) using enzymatic coupled reaction as described (Klingenberg, 1974). Extracellular organic acids were determined by high performance ionic chromatography (HPIC) using a Dionex Bio-LC500 apparatus as described previously (Groussac et al., 2000). Pi was measured in crude extract according to Bencini et al. (1983). For measuring intracellular glycerol, $20 \mathrm{ml}$ of cell sample were quickly filtrated onto $0.45 \mu \mathrm{m}$ nitrocellulose filter, washed once by $20 \mathrm{ml}$ of $-20{ }^{\circ} \mathrm{C} 60 \%$ methanol solution. The filters were immersed into $2 \mathrm{ml}$ of cold water and the tubes were put for $10 \mathrm{~min}$ in a water bath set at $90{ }^{\circ} \mathrm{C}$. After centrifugation of the cells debris at $10,000 \mathrm{~g}$ for $5 \mathrm{~min}$, glycerol was measured in the supernatant by a NADH-coupling enzymatic assay at $340 \mathrm{~nm}$. The reaction was done in $50 \mathrm{mM}$ TrisCl pH 7.4 containing $5 \mathrm{mM}$ $\mathrm{MgCl}_{2}, 1 \mathrm{mM} \mathrm{NAD}^{+}$and $1 \mathrm{U} \mathrm{ml}^{-1}$ glycerol-3-phosphate dehydrogenase (from Roche, Mannheim, Germany).

\section{Results}

\section{Construction of a glycerol hyper producer strain}

In a well-aerated glucose synthetic medium $\left(20 \mathrm{gl}^{-1}\right.$ glucose), the production of glycerol by the wild type yeast strain (CEN.PK2) reached a maximum of $2.0 \pm 0.4 \%$ of the glucose consumed (Table 3). This level of glycerol can be explained in part by the fact that yeast cells are endowed with other redox systems for maintaining the intracellular $\mathrm{NAD}^{+} / \mathrm{NADH}$ balance (Bakker et al., 2001), and also because the $\mathrm{NAD}^{+}$-dependent glycerol-3-P-dehydrogenase (GPD) that catalyzes the reduction of DHAP into glycerol3-P is rate-limiting for glycerol production (Cronwright et al., 2002; Larsson et al., 1993; Nevoigt and Stahl, 1996; Remize et al., 2003). Therefore, it was obvious to evaluate at first side effects of overproduction of this enzyme. To this end, the GPDI ORF was fused upstream of a strong $A D H 1$ promoter and this construct was integrated at the leu2 locus in the genome of the strain CEN.PK2. Table 3 shows that the glycerol yield in the GPDI-overexpressing strain was 10 -fold higher than in the wild type (Table 4) and this rise of production was in the same order of magnitude as the increase of glycerol-3-phosphate dehydrogenase activity in the transformed strain, which indicates that a large part of the flux control in glycerol pathway is hierarchical (ter Kuile and Westerhoff, 2001).

Increasing the availability of NADH is an additional mode that may favour glycerol production. This suggestion can be directly evaluated by deleting TPII encoding triose phosphate isomerase, since this genetic intervention should redirect half of the glucose molecule into the glycerol pathway (see Fig. 1). However, the downside of this intervention is the inability of a tpil $\Delta$ mutant to grow on a minimal glucose medium (Campagno et al., 1996; Overkamp et al., 2002) our data not shown), because of its inositol defective phenotype (Shi et al., 2005). This phenotype was shown to be a consequence of the inhibition of the myo-inositol synthase encoded by INOI by high levels of DHAP accumulating in tpil $\Delta$ mutants. Interestingly, the overexpression of GPDI restored the growth of the tpil $\triangle$ mutant, although the maximal growth rate of the tpil $\triangle G P D 1$-overexpressing strain was still 4 times lower than that of the isogenic wild type. Taken together, this strategy yielded a glycerol producer strain that exhibited a glycerol production yield of $0.36 \mathrm{~g}$ (g glucose $)^{-1}$, similar to

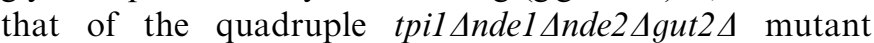

Table 3

Growth rate, yield of biomass and fermentation products in engineered strains for glycerol production obtained from shake flask cultures

\begin{tabular}{|c|c|c|c|c|c|c|}
\hline Strains & Genetic modification & Growth rate $\left(\mathrm{h}^{-1}\right)$ & Biomass yield & Ethanol & Glycerol & Acetate \\
\hline $\mathrm{HC} 13$ & GPD1 overexpression & $0.44 \pm 0.04$ & $10 \pm 0.3$ & $32 \pm 3.0$ & $19.3 \pm 1.4$ & $5 \pm 0.5$ \\
\hline $\mathrm{HC} 17$ & $A D H 1$ deletion & $0.26 \pm 0.03$ & $11 \pm 0.5$ & $29 \pm 2.0$ & $19 \pm 2.5$ & $13 \pm 0.8$ \\
\hline $\mathrm{HC} 23$ & $G P D 1$ and $A L D 3$ overexpression & $0.39 \pm 0.03$ & $10 \pm 1.3$ & $33 \pm 1.0$ & $18.5 \pm 2.0$ & $5 \pm 0.6$ \\
\hline $\mathrm{HC} 30$ & GPD1 and $A L D 3$ overexpression and $T P I 1$ deletion & $0.13 \pm 0.03$ & $9 \pm 1.4$ & $19 \pm 2.0$ & $42 \pm 2.5$ & $8 \pm 0.4$ \\
\hline FM62 & $G P D 1, A L D 3$ and $F P S 1$ overexpression, $T P I 1+A D H 1$ deletion & $0.12 \pm 0.02$ & $9 \pm 1.4$ & $12 \pm 2.8$ & $46 \pm 1.7$ & nd \\
\hline
\end{tabular}

Growth was carried out in SD medium at pH 5.0 as described in Material and methods. Yield for each metabolites was determined at the time of the complete consumption of glucose from the medium and it is expressed as ' $\mathrm{g}$ product per $100 \mathrm{~g}$ glucose'. Values of yield are the mean \pm standard deviation from four independent experiments. nd $=$ not determined. 
reported earlier (Overkamp et al., 2002). However, this latter engineered strain as analysed prior to its adaptation to high sugar concentration, had a maximal growth rate 2 times lower than that of our tpil $\Delta$ mutant overexpressing GPD1 strain $\left(0.045 \mathrm{~h}^{-1}\right.$ versus $\left.0.10 \mathrm{~h}^{-1}\right)$.

Another option for increasing glycerol production was to remove the major alcohol dehydrogenase encoded by $A D H 1$, since this genetic intervention should also favour NADH reoxidation through glycerol formation (Fig. 1). The glycerol yield of the adhl mutant was found to be comparable to that of the GPDI-overexpressing strain. Moreover, the deletion of $A D H 1$ resulted in a 2-fold reduction of the maximal growth rate (Table 3). This reduction could be due to an insufficient rate of reoxidation of NADH by the native GPDI and/or to an accumulation of acetaldehyde, a potential toxic molecule for growth (Michnick et al., 1997). An argument in favour of the first suggestion was the fact that the adhl $\Delta$ mutant recovered the wild type growth rate upon overexpression of GPD1 $\left(0.40 \mathrm{~h}^{-1}\right.$ for the adh1 $\triangle G P D 1$ versus $0.44 \mathrm{~h}^{-1}$ for the wild type, Table 3). Nevertheless, the yield of glycerol from glucose was not improved in the adhl mutant that overexpressed of GPD1 (data not shown). A further possibility to enhance NADH availability could be to promote the conversion of pyruvate to acetate via pyruvate decarboxylase and $\mathrm{NAD}^{+}$-dependent aldehyde dehydrogenase-driven reactions (see Fig. 1). ALD3 which encodes a cytosolic $\mathrm{NAD}^{+}$-dependent aldehyde dehydrogenase reported to be implicated in alanine biosynthesis (White et al., 2003) was overexpressed with the expectation that this enzyme could act on acetaldelyde to form acetate. Contrary to expectation, this genetic construct only slightly increased the glycerol yield when integrated in a tpil $\triangle G P D 1$ mutant to yield $\mathrm{HC} 30$ strain, and had no effect in a GPD1-overexpressing strain. We therefore evaluated the combination of GPDI overexpression with the deletion of TPII and $A D H 1$ on glycerol production.
The resulting engineered $\mathrm{HC} 32$ strain exhibited a glycerol yield of $0.46 \mathrm{~g}$ (g glucose $)^{-1}$, which equalled the yield of the engineered $S$. cerevisiae strain lacking pyruvate decarboxylase activity and mitochondrial-localized NADH dehydrogenases ( $p d c 1 \Delta$ pdc2 $\Delta$ pdc5 4 ndel $\Delta$ nde2 $\Delta$ gut $2 \Delta$ ) constructed by Geertman et al. (2006). However, a significant production of ethanol of about $0.1 \mathrm{~g}(\mathrm{~g} \text { glucose })^{-1}$ still took place in these engineered strains, which could be attributed to the $5 \%$ residual alcohol dehydrogenase activity from expressed minor $A D H$ genes (Drewke et al., 1990). The last step was to overexpress $A L D 3$ in $\mathrm{HC} 32$ strain. Because in shake flasks cultures, we observed a weak but reproducible $5-10 \%$ increase in glycerol production due to this gene overexpression (data not shown) that was not really confirmed in controlled batch cultures, we were inclined to use that latter engineered strain for further characterization of glucose fermentation and for evaluating the consequences of the genetic strategy by a comparative metabolic and genomic study to the isogenic wild type strain.

\section{Macrokinetic analysis of engineered strains for glycerol overproduction}

Fig. 2 shows the growth curve and by-products formation of wild type, HC16 (tpil $\triangle G P D 1$ ) and $\mathrm{HC} 42$ (tpil $\triangle$ adh1 $\triangle A L D 3$ GPD1) strains growing on a glucose minimal medium in $\mathrm{pH}$-controlled batch fermentors. Since the three cultures were inoculated with the same amount of cell biomass, it could be seen that the time required for complete consumption of glucose by the engineered strains ( $\mathrm{HC} 16$ and $\mathrm{HC} 42$ strains) was greater than that of the isogenic wild type. However, the $\mathrm{HC} 42$ was able to ferment glucose more rapidly than $\mathrm{HC16}$, suggesting a regain of growth efficiency as a consequence of a better management of NADH regeneration due to the loss of $A D H 1$ function and/or to the increased expression of $A L D 3$ in HC42. Also,

Table 4

Enzyme activity in engineered strains for glycerol production

Enzyme activity $\left(\mathrm{nmol} \mathrm{min}^{-1}\right.$. mg protein)

\begin{tabular}{|c|c|c|c|c|c|c|c|c|c|c|c|}
\hline \multirow[t]{2}{*}{ Strains } & \multirow[t]{2}{*}{ G6PD } & \multirow[t]{2}{*}{ PGI } & \multirow[t]{2}{*}{ GPD } & \multirow[t]{2}{*}{ GPP } & \multicolumn{4}{|l|}{ GDH } & \multirow[t]{2}{*}{ ADH } & \multicolumn{2}{|l|}{ “ALD” } \\
\hline & & & & & $\mathrm{NAD}^{+}$ & $\mathrm{NADP}^{+}$ & $\mathrm{NAD}^{+}$ & $\mathrm{NADP}^{+}$ & & $\mathrm{NAD}^{+}$ & $\mathrm{NADP}^{+}$ \\
\hline CEN.PK2 & $312 \pm 3.1$ & $1580 \pm 214$ & $23 \pm 4$ & $450 \pm 30$ & $13.6 \pm 0.7$ & $13.0 \pm 1.7$ & 21.2 & 19.3 & $4610 \pm 10$ & $57.3 \pm 6.4$ & $149 \pm 6.0$ \\
\hline $\mathrm{HC} 13$ & nd & $1318 \pm 69$ & $360 \pm 24$ & $440 \pm 30$ & $2.6 \pm 0.3$ & bd & nd & nd & $3762 \pm 50$ & nd & $115 \pm 3.6$ \\
\hline $\mathrm{HC} 16$ & $214 \pm 5.4$ & $1831 \pm 109$ & $387 \pm 12$ & $660 \pm 34$ & 9.0 & bd & nd & nd & $4449 \pm 43$ & $15 \pm 3.0$ & $46 \pm 3.1$ \\
\hline HC30 & $302 \pm 3$ & $2620 \pm 94$ & $318 \pm 23$ & $840 \pm 30$ & $10.9 \pm 0.5$ & 8.5 & nd & nd & nd & $18 \pm 0.4$ & $103 \pm 3.5$ \\
\hline $\mathrm{HC} 32$ & $238 \pm 8$ & $1681 \pm 24$ & $259 \pm 11$ & $530 \pm 40$ & $8.1 \pm 0.4$ & 12.8 & $53.6 \pm 1$ & $31 \pm 2.3$ & $274 \pm 23$ & $12 \pm 2$ & $177 \pm 3.0$ \\
\hline $\mathrm{HC} 42$ & $267 \pm 14$ & $1821 \pm 62$ & $357 \pm 78$ & $680 \pm 40$ & $10.8 \pm 4.1$ & bd & $31.8 \pm 10$ & $38 \pm 2.4$ & $358 \pm 34$ & $15 \pm 3$ & $166 \pm 16.4$ \\
\hline
\end{tabular}

The values are the mean $\pm \mathrm{SD}$ of three independent experiments. $\mathrm{G} 6 \mathrm{PD}=$ glucose-6-P dehydrogenase; PGI $=$ glucose-6-phosphate isomerase; GPD $=$ glycerol-3-P dehydrogenase; GPP $=$ glycerol-3-P phosphatase; $\mathrm{GDH}=\mathrm{NAD}^{+}$or NADP-dependent glycerol dehydrogenase; $\mathrm{ADH}=$ alcohol dehydrogenase; $\mathrm{ALD}=$ acetaldehyde dehydrogenase. $\mathrm{nd}=$ not determined; $\mathrm{bd}=$ below detection. 

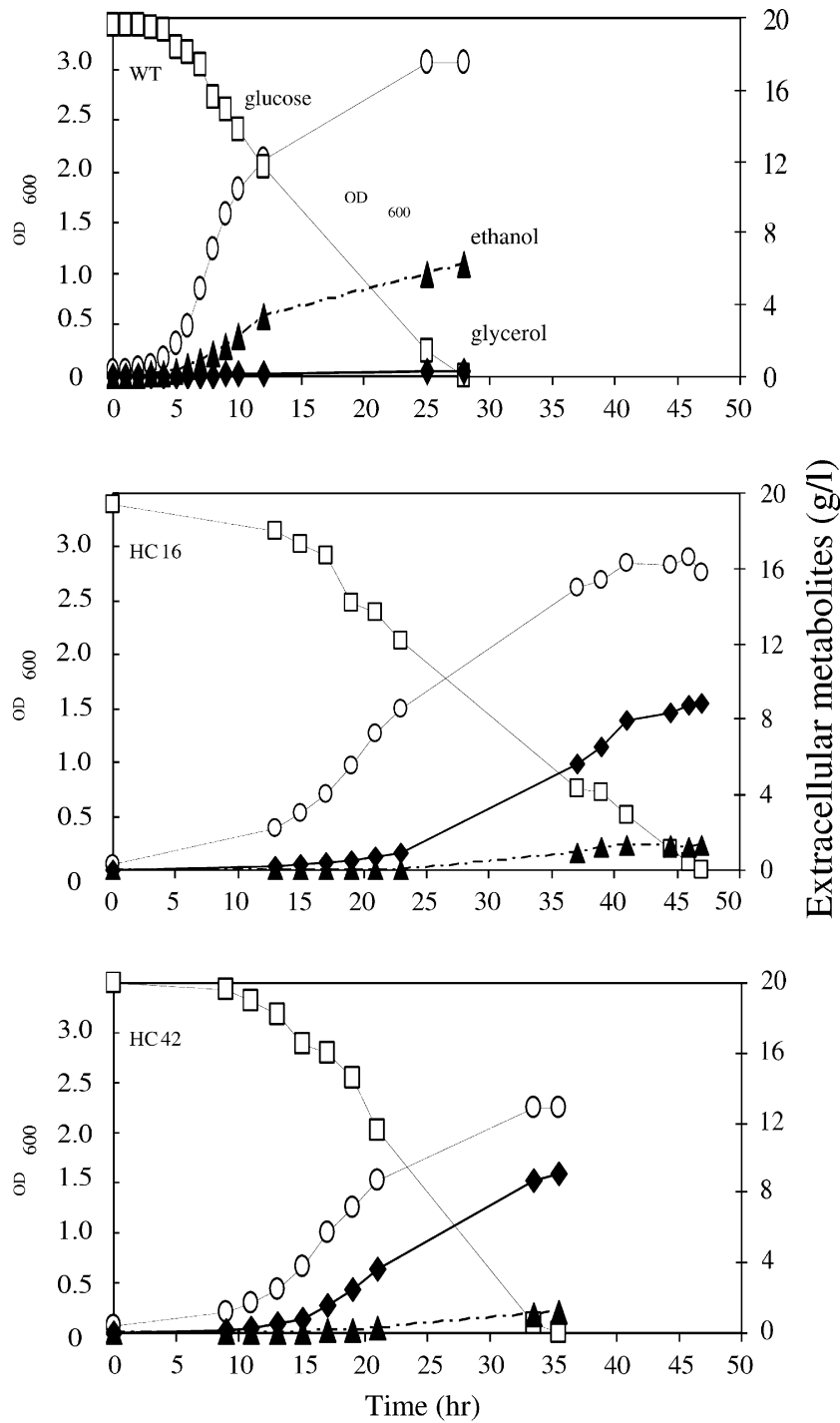

Fig. 2. Macrokinetics behaviour of wild type and engineered strains during glycerol production in aerobic batch cultures on glucose. Growth was carried out at $30^{\circ} \mathrm{C}$ in synthetic mineral medium containing $20 \mathrm{gl}^{-1}$ glucose and supplemented with autotrophy requirements. and as expected, glycerol became the major fermentation product in the engineered strains. A summary of the kinetic fermentation parameters of wild type and engineered strains is reported in Table 5. As already noticed in Fig. 2, a consequence of engineering yeast for high glycerol production was a significant reduction in the growth rate $\left(\mu_{\max }\right)$. However, the $\mu_{\max }$ of the multi-engineered strain HC42 was $\approx 30 \%$ higher than that of HC16. This relative better growth rate was associated with a glucose consumption and a glycerol production rates that were, respectively, $17 \%$ and $55 \%$ higher than in HC16 (Table 5). Moreover, the glycerol yield in $\mathrm{HC} 42$ was $10 \%$ higher than in $\mathrm{HC} 16$

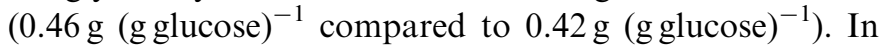
addition, it was not surprising that an elevated production of glycerol was accompanied by a reduction of biomass yield since production of this metabolite is an ATP consuming process. Table 6 gives the yield of major byproducts identified at the end of the fermentation when glucose has been completely consumed. Major changes in yield were obtained for ethanol and glycerol. Besides these metabolites, acetaldehyde and acetoin were not dramatically increased in the HC42 after deletion of $A D H 1$, probably because the aeration process of the bioreactor stripped away acetaldehyde as soon as it was produced. Also, levels of acetate barely changed in HC42 strain that overexpressed $A L D 3$ encoding a cytosolic $\mathrm{NAD}^{+}$-aldehyde dehydrogenase. This lack of effects on acetate levels indicated that this aldehyde dehydrogenase, even present at high levels in the cells, was unable to act on acetaldehyde. Taking into account these by-products, we evaluated the carbon balance. This balance was close to $100 \%$ for the wild type, consistent with the fact that under this growth condition, the major fermentation products were ethanol, glycerol, acetate and biomass. In contrast, the carbon balance was around 90-92 in the two engineered strains. Possible reasons for these values may be an underestimation of acetaldehyde, as well as an underestimation of $\mathrm{CO}_{2}$ production by a higher respiratory activity of the engineered strains, as inferred from transcriptomic data (see below).

Table 5

Specific rates of growth, glycerol and ethanol production and glucose consumption of wild type and engineered strains for glycerol production

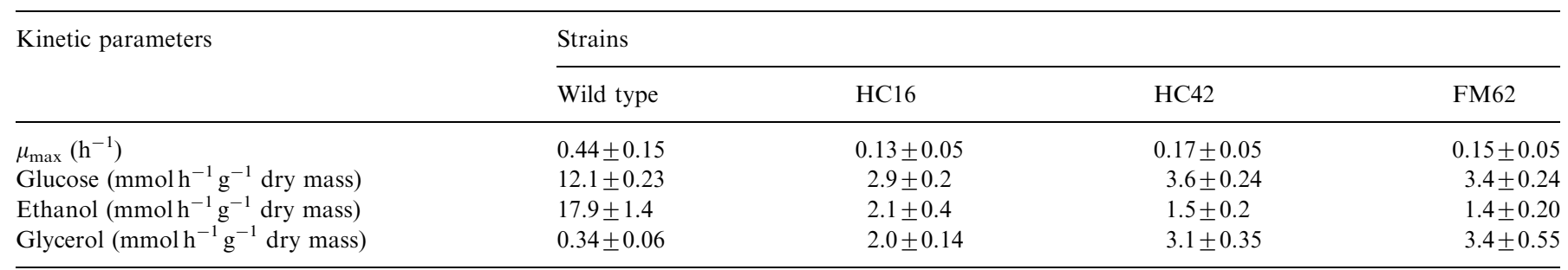

Yeast strains were cultivated in aerobic batch cultures on a SD medium containing $20 \mathrm{gl}^{-1}$ glucose. The maximal specific rates were calculated as follows: production of biomass and metabolites along the growth were fitted to a third order polynomic function with $R^{2}>0.995$. At time interval during the exponential growth phase, the derivative of this equation for glucose consumption, glycerol and ethanol production was determined and divided by that corresponding to the biomass production. The data are the mean of two independent cultures including standard deviation. 
Table 6

Yield of biomass and fermentation products during batch culture of the isogenic wild type and two engineered strains for glycerol production

\begin{tabular}{lllc}
\hline Fermentation products & \multicolumn{3}{l}{ Strains } \\
\cline { 2 - 4 } & Wild type & HC16 & HC42 \\
\hline Biomass & $12.5 \pm 1.30$ & $9.98 \pm 1.5$ & $8.7 \pm 1.1$ \\
Ethanol & $40.4 \pm 2.7$ & $17.4 \pm 0.7$ & $11.6 \pm 0.7$ \\
Glycerol & $1.50 \pm 0.20$ & $41.8 \pm 3.3$ & $45.8 \pm 2.5$ \\
Acetate & $4.90 \pm 0.34$ & $1.98 \pm 0.7$ & $2.80 \pm 0.7$ \\
Pyruvate & $0.45 \pm 0.10$ & $0.35 \pm 0.05$ & $0.66 \pm 0.10$ \\
Acetaldehyde & $0.40 \pm 0.10$ & $0.21 \pm 0.05$ & $2.9 \pm 0.07$ \\
Acetoine & $0.65 \pm 0.07$ & $0.27 \pm 0.04$ & $2.1 \pm 0.20$ \\
Succinate & bd & $0.25 \pm 0.04$ & $0.55 \pm 0.14$ \\
Citrate & bd & 0.14 & $0.68 \pm 0.10$ \\
Carbon balance $(\%)$ & 99.9 & 92.2 & 90 \\
\hline
\end{tabular}

The fermentation was carried out in controlled batch reactor at $\mathrm{pH} 5.0$ in SD medium. Glucose and fermentation products were monitored along the growth. The yield is calculated by taking levels of biomass and metabolites that were produced at the time of the complete consumption of glucose, and is expressed in $\mathrm{g}$ per $100 \mathrm{~g}$ glucose. Values reported are the mean of two independent experiments including standard deviation. $\mathrm{CO}_{2}$ was calculated from ethanol production. $b d=$ below detection.

\section{Glycerol efux is rate limiting in glycero Ihyperproducer strains}

The construction of engineered strains for glycerol production raised at least two metabolic issues, one of them is developed in this section. The first challenge was to restore growth of a tpil $\Delta$ mutant on glucose. The failure of this mutant to grow on glucose was recently attributed to its inositol-less phenotype. This auxotrophy was explained by a potent inhibition of the myo-inositol- 6- phosphate (MIP) synthase by high levels DHAP and glycerol-3-P present in mutants defective in triose-phosphate isomerase activity. Accordingly, the growth on a glucose synthetic media could be restored in the presence of inositol (Shi et al., 2005; and our unpublished data). Here, we found that the growth on glucose of our tpil $\triangle$ mutant was recovered upon overexpression of GPD1. As shown in Table 7, this growth recovery can be in part explain by a significant decrease (3- to 4- fold) of DHAP and glycerol-3-P in a tpil $\triangle$ mutant that overexpressed GPDI and in a tpil $\triangle$ GPD1 strain that was also deleted for $A D H 1$ (Table 7). However, the fact that levels of DHAP and glycerol 3-P did not return to levels in wild type cells suggested that the glycerol flux in the engineered strains was no longer limited at the level of glycerol-3-P dehydrogenase activity (Cronwright et al., 2002; Remize et al., 2001). Evidence that the glycerol export became the major bottleneck in glycerol production was given by the finding that $\mathrm{HC} 16$ and $\mathrm{HC} 42$ accumulated 7-10 times more intracellular glycerol than the isogenic wild type (Table 7). Taking into account the enzymatic and metabolic data reported in Tables 4, 5 and 7 , and using the kinetic parameters of GPD and GPP reported by Cronwright et al. (2002), we roughly estimated
Table 7

Metabolites levels in strains engineered for glycerol production

\begin{tabular}{|c|c|c|c|c|c|}
\hline $\begin{array}{l}\text { Metabolites } \\
\left(\mu \text { mol g }^{-1} \text { dry mass }\right)\end{array}$ & Wild type & $\mathrm{HC} 14$ & $\mathrm{HC} 16$ & $\mathrm{HC} 42$ & FM62 \\
\hline DHAP & $1.89 \pm 0.50$ & $35.7 \pm 8.20$ & $10.90 \pm 4.30$ & $8.9 \pm 3.30$ & nd \\
\hline Glycerol-3-P & $0.19 \pm 0.06$ & $4.10 \pm 0.90$ & $3.57 \pm 1.0$ & $1.60 \pm 0.70$ & nd \\
\hline Glycerol $_{\text {in }}$ & $23 \pm 5.50$ & $59 \pm 14.6$ & $160 \pm 55$ & $212 \pm 36$ & $115 \pm$ \\
\hline $\mathrm{NAD}^{+}$ & $2.75 \pm 0.79$ & $3.08 \pm 0.38$ & $3.52 \pm 0.64$ & $2.95 \pm 0.59$ & nd \\
\hline $\mathrm{NADH}$ & $0.167 \pm 0.08$ & $0.031 \pm 0.008$ & $0.039 \pm 0.01$ & $0.038 \pm 0.012$ & nd \\
\hline $\mathrm{NAD}^{+} / \mathrm{NADH}$ & 16.7 & 97.7 & 79 & $77-$ & nd \\
\hline ATP & $3.85 \pm 0.35$ & $1.83 \pm 0.55$ & $1.97 \pm 0.28$ & $2.65 \pm 0.30$ & nd \\
\hline ADP & $1.07 \pm 0.12$ & $1.46 \pm 0.33$ & $1.49 \pm 0.34$ & $1.85 \pm 0.07$ & nd \\
\hline AMP & $0.165 \pm 0.12$ & $1.67 \pm 0.24$ & $1.23 \pm 0.09$ & $0.81 \pm 0.15$ & nd \\
\hline $\mathrm{AC}$ & 0.86 & 0.51 & 0.58 & 0.64 & nd \\
\hline
\end{tabular}

Yeast strains were cultivated in SD medium supplemented with $55 \mu \mathrm{M}$ inositol (only for HC14). Metabolites were measured in samples taken at the exponential phase of growth $\left(\mathrm{OD}_{600}\right.$ around 1.0). Values reported are the mean \pm standard deviation of four independent experiments. AC $($ adenylate charge $)=\left(\mathrm{ATP}+\frac{1}{2} \mathrm{ADP}\right) / \mathrm{AMP}+\mathrm{ADP}+\mathrm{ATP}$. nd: not determined.

the flux control coefficient for these three enzymatic steps of the glycerol pathway to be around $0.25-0.30,0.0-0.10$ and $0.65-0.70$ for GPD, GPP and glycerol efflux, respectively. Consistent with the fact that the rate-limiting step was shifted at the level of the glycerol efflux, overexpression of FPS1 encoding the major glycerol facilitator (Luyten et al., 1995) in HC42 (to yield FM62 strain) resulted in a 2-fold reduction of intracellular glycerol and in a $10 \%$ increase in glycerol productivity (Tables 5 and 7).

\section{Effects of genetic engineering on NADH availability for glycerol production}

A second metabolic question was to evaluate effects of these molecular manipulations on levels of $\mathrm{NAD}^{+}$and $\mathrm{NADH}$, as it is thought that availability of NADH is determinant for glycerol production (Cambon et al., 2006; Geertman et al., 2006; Overkamp et al., 2002). As indicated in Table 7, the genetic manipulation of yeast for glycerol production led to a significant decrease in $\mathrm{NADH} / \mathrm{NAD}^{+}$ ratio, which was essentially attributed to a 4- to 5- fold reduction in NADH pools. This low $\mathrm{NADH} / \mathrm{NAD}^{+}$ratio together may be somehow inhibitory for glycerol production since $\mathrm{NAD}^{+}$acts as a competitive inhibitor of NADH on Gpdlp activity (Albertyn et al., 1992). Levels of ATP were also lower in engineered strains, and especially in the tpild mutant. As ADP and AMP were also measured, this allowed to evaluate the adenylate charge (AC) of the cells according to the Atkinson' equation (Chapman and Atkinson, 1977). The wild type cells exhibited an AC value of 0.86 , which is commonly obtained under normal growth conditions. In contrast, this value dropped to 0.54 in a tpild mutant, consistent with its poor growth on glucose. Value of AC was slightly increased to 0.58 in $\mathrm{HC} 16$ and 
0.64 in HC42 strains. Although lower than in wild type cells, these $\mathrm{AC}$ values were not significantly low to account for carbon metabolism constraints in the engineered strains.

Other biochemical effects of our genetic engineering strategy needed some comments. The overexpression of $A L D 3$ did not result in an increase of acetate, which reinforced the recent finding that this $A L D 3$-encoding aldehyde dehydrogenase is really specific in the conversion of 3-aminopropanal to $\beta$-alanine (White et al., 2003). The engineered HC42 (tpil $\triangle$ adhl GPDI) strain was found to contain 5 times more alcohol dehydrogenase activity than the adh1 $\Delta$ mutant (HC17 mutant, Table 4), suggesting a partial release of the glucose-repressed alcohol dehydrogenases. Furthermore, the putative $\mathrm{NAD}^{+}$-dependent glycerol dehydrogenase activity that converts glycerol into dihydroxyacetone (DHA) increased by about 2 -fold in HC32 and HC42 strains. Altogether, these enzymatic changes could penalize glycerol production.

\section{A genome-wide transcriptomic analysis of glycerol hyperproducer} strains

To evaluate the effects of molecular manipulations for glycerol production at the genomic scale, we carried out a transcriptomic analysis of the wild type and the multiengineered $\mathrm{HC} 42$ strains cultivated in well-aerated batch reactors on a $20 \mathrm{gl}^{-1}$ glucose medium. Samples were taken early in the log phase, at the maximal growth rate. The culture was repeated 4 times to obtain four independent data sets for statistical analysis (see Material and methods for other details). Using two filtering criteria (ratio $>1.5$ or $<-1.5$, and $p$ value $<0.05$ ), a total of 384 differentially expressed genes between $\mathrm{HC} 42$ and CEN.PK2 $\alpha$ were retained, from which 184 were downregulated and 200 were upregulated (see the complete data sets at http:// biopuce.insa-toulouse.fr/supdata/glycerolgenomics). As a first step for data interpretation, the differentially expressed genes were grouped into 13 functional categories according to the MIPS nomenclature (Mewes et al., 1997; Ruepp et al., 2004). The procedure was to express the number of up- and down-regulated transcripts in each of the categories as a percentage of the total differentially expressed genes and to compare this functional classification with the functional catalogues of genes described in MIPS. This procedure has the advantage to easily identify the major gene expression remodelling on a genomic scale. As it can be seen in Fig. 3, the multiple genetic modifications introduced in CEN.PK2 to reorient the carbon flux towards glycerol production resulted in significant enrichment in transcripts of genes that belong to carbon and energy metabolism and to protein synthesis, and in a lesser extent, to ionic homeostasis and cellular communication. In contrast, genes encoding products involved in transcription, cell biogenesis, cell cycle and DNA processing were significantly under-represented. This was further consolidated by GO analysis of the expression data, which authorizes a better identification of the biological processes and cellular components. This analysis revealed that the biological processes altered in the engineered strain concerned cell growth, energy metabolism, ribosomal protein synthesis and ribosomal biogenesis (see Fig S1, in Supplementary data). A closer inspection of genes belonging to the ribosomal protein synthesis and ribosome biogenesis showed that $>90 \%$ of these genes were downregulated, which was consistent with a dramatic reduction of the growth rate of the engineered strain (Table S1, in Supplementary data).

Other relevant transcriptomic information could be exploited from the list of upregulated genes, whose most of them belong to carbon and energy metabolism (Table 8). As expected, ALD3 and GPD1 were overexpressed (respectively 6- and 4-fold), but also GPD2 which encodes the second GPD isoform. The upregulation of INOI (3.3fold) encoding MIP synthase as well as GIT1 (1.7-fold) encoding a permease for uptake of glycerophosphoinositol was consistent with the inositol defect induced by deletion of TPII. This list also contained several genes encoding proteins with $\mathrm{NAD}^{+} / \mathrm{NADP}^{+}$binding function (NDII, PDH1, ALD4, ARA1, GDH2, IDH1, ALD2, ETR1). The "cellular transport and facilitation" category stood as another important functional category that was significantly perturbed by genetic engineering for glycerol production (Table S2, in Supplementary data). Among others, transcript levels of JEN1 which encodes a glucoserepressible lactate/pyruvate permease (Bojunga and Entian, 1999; Casal et al., 1999) was about 2-fold higher in HC42. We also noticed an activation of PHOS4 encoding the high affinity phosphate permease (Bun-ya et al., 1991), which was expected due to the regulatory link between inositol and phosphate metabolism (Almaguer et al., 2003), and a 2 -fold upregulation of $S L T 1$ encoding the glycerol $/ \mathrm{H}^{+}$symporter (Ferreira et al., 2005). The latter activation may be a consequence of the increased osmotic potential of the cytoplasm due to intracellular accumulation of glycerol.

When the genes listed in Table 8 were clustered according to a regulation by a common transcriptional regulation using YEAStract software (Teixeira et al., 2006), $30 \%$ of them were found to be co-regulated by the HAP $2 /$ $3 / 4 / 5$ complex, the major transcriptional activator of respiratory genes expression (see Table S2 in Supplementary data). This result was indicative of a partial derepression of the glucose-repressed respiratory activity in the glycerol producer strain. Accordingly, the expression level of some genes known to be repressed by glucose (i.e., $C O X 3$; SDH2, SDH3, CYT1, ADR1, etc., see Table 8) were upregulated in the engineered strain.

\section{Discussion}

The purpose of this work was to explore an alternative engineering strategy for glycerol production than those previously reported (Campagno et al., 1996; Geertman 


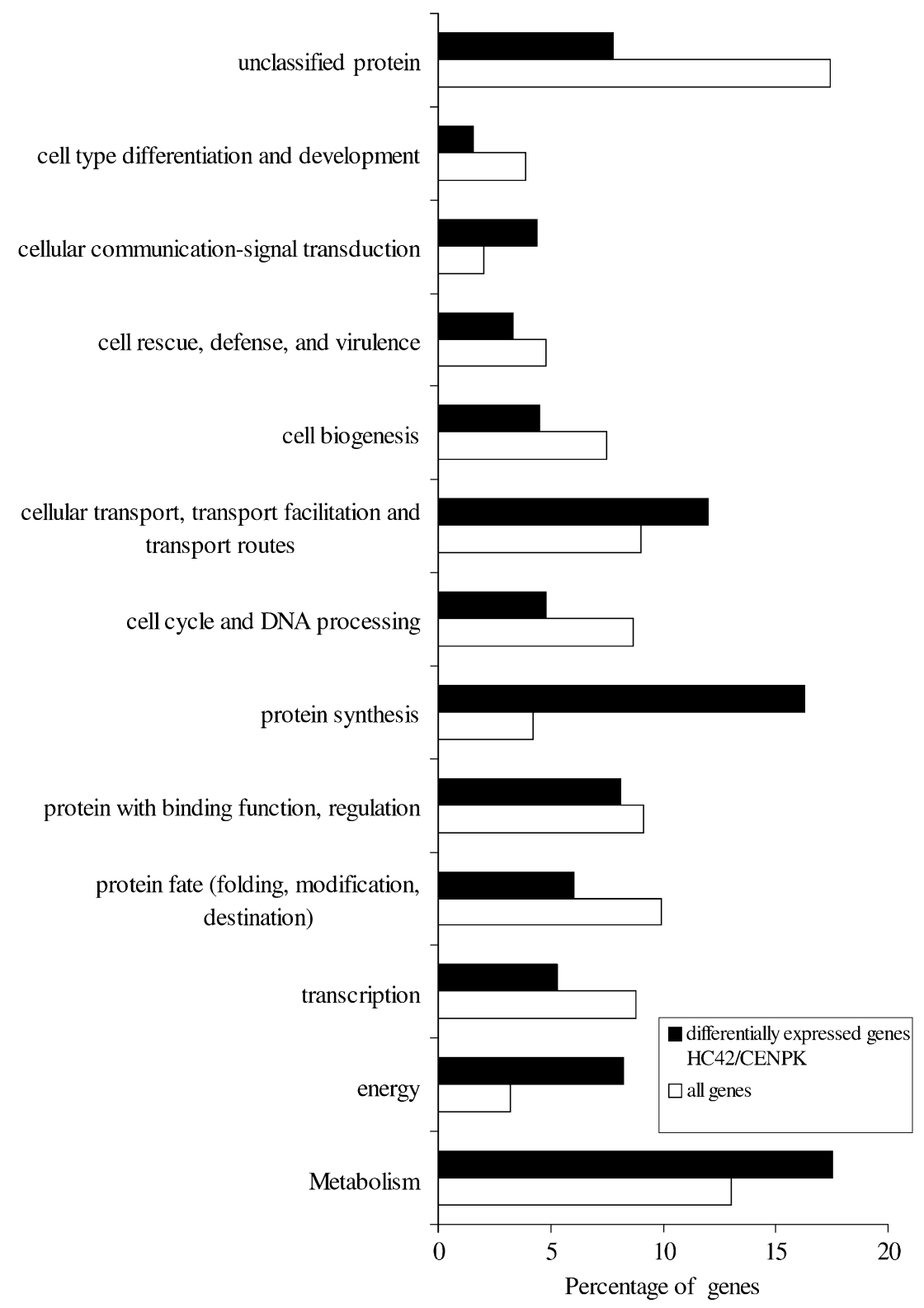

Fig. 3. Distribution of the differentially expressed genes in the engineered HC42 strain according to the functional categories. Open histograms represent the functional catalogue of 6200 genes according to the MIPS classification. Solid histograms represent the distribution of the 384 differentially expressed genes from HC42 strain versus wild type in the functional categories of MIPS. The percentage of each category was calculated as the number of up- and down-regulated genes from each of the 13 categories divided by the total number of differentially expressed genes in engineered strain.

et al., 2006; Nevoigt and Stahl, 1996; Overkamp et al., 2002; Remize et al., 1999) and to evaluate carefully the consequences of this strategy at the metabolic and genomic levels. Our plan involved the overexpression of GPDI and $A L D 3$ together with deletion of TPII and $A D H 1$. This strategy yielded an engineered strain with a glycerol production, which in terms of final yield and maximal productivity rate, was significantly better than that obtained by Overkamp et al. (2002) using the same strain

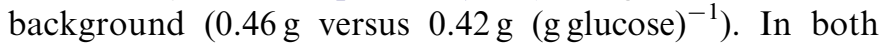
cases, a tpil $\Delta$ strain has been used. While Overkamp and coworkers adopted a strategy that promoted glycerol production by preventing regeneration of NADH by mitochondrial NADH dehydrogenases through deletion of NDE1, NDE2 and GUT2 in the tpild strain, our approach was more effective for glycerol productivity and growth recovery for at least two reasons. Firstly, Gpdlp is rate limiting in glycerol synthesis, as determined by Metabolic Control analysis (Cronwright et al., 2002). Thus, its overproduction should stimulate the carbon flux in this pathway. Secondly, the inability of a tpil null mutant to grow on glucose has been recently explained by 
Table 8

List of upregulated genes in HC42 strain versus CEN.PK2 $\alpha$ that belong to metabolism and energy categories

\begin{tabular}{|c|c|c|c|}
\hline ORF & Gene & Fold change & Description \\
\hline YMR169C & $A L D 3$ & 6.56 & Cytoplasmic aldehyde dehydrogenase uses NAD + as the preferred coenzyme \\
\hline$Y D L 022 \mathrm{~W}$ & GPD1 & 4.14 & NAD-dependent glycerol-3-phosphate dehydrogenase, key enzyme of glycerol synthesis \\
\hline YLL041C & $\mathrm{SDH} 2$ & 3.58 & Iron-sulfur protein subunit of succinate dehydrogenase \\
\hline YML120C & NDI1 & 3.37 & NADH:ubiquinone oxidoreductase \\
\hline YJL153C & INOI & 3.27 & Inositol 1-phosphate synthase \\
\hline YFL014W & HSP12 & 3.1 & Plasma membrane-localized protein induced by heat shock \\
\hline YPR002W & PDH1 & 2.81 & Mitochondrial protein that participates in respiration \\
\hline YPL058C & PDR12 & 2.39 & Plasma membrane weak-acid-inducible ATP-binding cassette (ABC) transporter \\
\hline YOL096C & $\mathrm{COQ3}$ & 2.29 & O-methyltransferase, component of a mitochondrial ubiquinone-synthesizing complex \\
\hline YDR216W & $A D R 1$ & 2.21 & Carbon source-responsive zinc-finger transcription factor \\
\hline YBR002C & RER2 & 2.13 & Cis-prenyltransferase involved in dolichol synthesis \\
\hline$Y B L 015 \mathrm{~W}$ & ACH1 & 2.1 & Acetyl-coA hydrolase \\
\hline YCL004 W & PGS1 & 2.09 & Phosphatidylglycerolphosphate synthase \\
\hline YOL059W & GPD2 & 2.08 & NAD-dependent glycerol-3-phosphate dehydrogenase, homolog of Gpdlp \\
\hline YOR065 W & CYT1 & 1.98 & Cytochrome $\mathrm{cl}$, component of the mitochondrial respiratory chain \\
\hline Q0080 & $A A P 1$ & 1.98 & Subunit 8 of the F0 sector of mitochondrial inner membrane F1F0 ATP synthase \\
\hline YOR374W & $A L D 4$ & 1.95 & Mitochondrial aldehyde dehydrogenase, required for conversion of acetaldehyde to acetate \\
\hline$Y D L 215 C$ & $G D H 2$ & 1.92 & NAD $(+)$-dependent glutamate dehydrogenase, degrades glutamate to ammonia and alpha-ketoglutarate \\
\hline YBR149W & ARA1 & 1.91 & Large subunit of NADP + dependent arabinose dehydrogenase \\
\hline YKL093W & $M B R 1$ & 1.9 & Protein involved in mitochondrial functions and stress response \\
\hline YDR298C & ATP5 & 1.88 & Subunit 5 of the stator stalk of mitochondrial F1F0 ATP synthase, (oligomycin sensitivity-conferring protein \\
\hline YGL191W & COX13 & 1.83 & Subunit VIa of cytochrome $c$ oxidase \\
\hline YJR121 W & ATP2 & 1.81 & Beta subunit of the F1 sector of mitochondrial F1F0 ATP synthase \\
\hline YJR034W & PET191 & 1.78 & Protein required for assembly of cytochrome $c$ oxidase \\
\hline YHR001W & OSH7 & 1.76 & Member of an oxysterol-binding protein family with seven members in $S$. cerevisiae \\
\hline YGR161C & RTS3 & 1.76 & Putative component of the protein phosphatase type $2 \mathrm{~A}$ complex \\
\hline YHR073W & $\mathrm{OSH} 3$ & 1.74 & Member of an oxysterol-binding protein family with seven members in $S$. cerevisiae \\
\hline YNL037C & IDH1 & 1.73 & Subunit of mitochondrial NAD(+)-dependent isocitrate dehydrogenase \\
\hline YLR295C & ATP14 & 1.72 & Subunit $h$ of the F0 sector of mitochondrial F1F0 ATP synthase \\
\hline$Y M R 217 \mathrm{~W}$ & GUA1 & 1.71 & GMP synthase, enzyme that catalyzes the second step in the biosynthesis of GMP from inosine 5'-phosphate \\
\hline YELO24W & RIP1 & 1.69 & Ubiquinol-cytochrome- $c$ reductase \\
\hline$Y G L 205 \mathrm{~W}$ & POX1 & 1.67 & Fatty-acyl coenzyme A oxidase, localized to the peroxisomal matrix \\
\hline$Y K L 141 \mathrm{~W}$ & $S D H 3$ & 1.67 & Cytochrome b subunit of succinate dehydrogenase \\
\hline YBR039W & ATP3 & 1.67 & Gamma subunit of the F1 sector of mitochondrial F1F0 ATP synthase \\
\hline YOR377W & ATF1 & 1.64 & Alcohol acetyltransferase with potential roles in lipid and sterol metabolism \\
\hline YPL262W & FUM1 & 1.63 & Fumarase, converts fumaric acid to L-malic acid in the TCA cycle \\
\hline YBR093C & PHO5 & 1.59 & Repressible acid phosphatase \\
\hline YDR074W & TPS2 & 1.59 & Phosphatase subunit of the trehalose-6-phosphate synthase/phosphatase complex \\
\hline YMR170C & $A L D 2$ & 1.57 & Cytoplasmic aldehyde dehydrogenase \\
\hline YML081CA & ATP18 & 1.56 & Subunit of the mitochondrial F1F0 ATP synthase; termed subunit $I$ or subunit $j$ \\
\hline YMR256C & $\mathrm{COX} 7$ & 1.55 & Subunit VII of cytochrome $c$ oxidase \\
\hline YBR026C & ETR1 & 1.54 & 2-enoyl thioester reductase \\
\hline YBR006W & $U G A 2$ & 1.54 & Succinate semialdehyde dehydrogenase involved in the utilization of gamma-aminobutyrate \\
\hline YBL033C & RIB1 & 1.53 & GTP cyclohydrolase II \\
\hline YDR178W & $\mathrm{SDH} 4$ & 1.53 & Membrane anchor subunit of succinate dehydrogenase \\
\hline YFL017C & GNA1 & 1.51 & Glucosamine-6-phosphate acetyltransferase \\
\hline YGL187C & $\mathrm{COX} 4$ & 1.5 & Subunit IV of cytochrome $c$ oxidase \\
\hline
\end{tabular}

its inositol-defective phenotype. This deficiency is due to the potent inhibition of the MIP synthase that catalyzes the synthesis of inositol-6-P from glucose-6-P, by DHAP and glycerol-3-P, which accumulate to very high levels in a tpil $\Delta$ mutant (Shi et al., 2005, this study). In this work, we showed that overexpression of GPDI significantly reduced DHAP and glycerol-3-P levels, and this reduction in these two metabolites is likely to be more potent than expected in a tpil $\triangle$ mutant defective of NDE1,NDE2 and GUT2.

However, levels of DHAP and glycerol-3-P in none of the engineered strains returned to those in a wild type strain, suggesting either a problem of NADH recycling or that the rate-limiting step of glycerol production was no longer at the level of GPD enzyme. The finding that engineered strains accumulated large amount of intracellular glycerol pointed to glycerol efflux as the major ratelimiting step in this process. This was confirmed by Metabolic Control analysis, which indicated that the value of the flux control coefficient at the glycerol efflux raised from almost 0.1 in normal condition to 0.70 in condition of hyperproduction of glycerol. Furthermore, intracellular accumulation of glycerol could be reduced 2- to 3-fold by overexpression of FPSI encoding the major glycerol facilitator (Luyten et al., 1995; Tamas et al., 1999). 
However, the fact that this overexpression had a minor effect on glycerol efflux could be explained by the recent finding that high intracellular content of glycerol can mediate its own closure (Karlgren et al., 2005). Alternatively, it is possible that other aquaglyceroporins are also needed for complete glycerol efflux (Luyten et al., 1995; Petterson et al., 2005). Accumulation of intracellular glycerol has been also reported to trigger a Hog1dependent osmoprotective pathway (Albertyn et al., 1994a; Siderius et al., 2000), which ultimately leads to a slow down of glycolysis and growth rate by a yet uncharacterized mechanism (Blomberg, 2000). Taken together, these effects may also explain why the overexpression of FPS1 resulted in a weak increase in glycerol productivity As a consequence, the removal of glycerol during its synthesis should be considered as a method to enhance its productivity.

Measurement of intracellular metabolites and coenzymes gave also some indications with respect to the metabolic constraints resulting from our engineering strategy. The huge increase of the $\mathrm{NAD}^{+} / \mathrm{NADH}$ associated with a reduction of ATP levels in the engineered strains indicated that the glycolytic activity in the yeast cells is restrained to its capacity to regenerate NADH. This result indicated that the glycerol production is not only limited by NADH availability but also to the capacity of the yeast cells to regenerate this coenzyme. This interpretation is consistent with the results of Geertman et al. (2006) that showed an increased glycerol production by co-feeding engineered strains with formate, as this latter substrate acts as a direct source of cytosolic NADH. However, taking into account that the rate limiting for glycerol synthesis in engineered strains is reset at the level of glycerol efflux, the co-feeding strategy is not sufficient to increase glycerol productivity.

Besides these metabolic bottlenecks, our transcriptomic analysis identified several secondary effects of the engineering strategy that may be counterproductive for glycerol production. A major trait was a significant upregulation of genes encoding $\mathrm{NAD}^{+} / \mathrm{NADP}^{+}$binding proteins and aldehyde dehydrogenases (i.e., $A R A 1, N D I 1, G D H 2$, $A L D 4, P D H 1, A L D 2$, etc). Additionally, the activity of $\mathrm{NAD}^{+}$-dependent glycerol dehydrogenase that converts glycerol into DHA was also increased. Altogether, these metabolic and transcriptomic perturbations could bring at least two negative effects on glycerol production, namely a possible withdrawing of NADH from the glycerol-3-P dehydrogenase enzyme and a potential wasteful glycerol cycle (Molin et al., 2003). A last but not the least significant effect of the metabolic engineering strategy was a partial derepression of the glucose-repressive genes that belong to the TCA cycle and respiratory activity in the glycerol hyperproducer $\mathrm{HC} 42$ strain, which supports the notion that the repression of these cellular functions are dependent on the rate of glucose assimilation (Blank and Sauer, 2004).

To summarize, we successfully generated a high glycerol producer strain that exhibits a glycerol yield $(0.46 \mathrm{~g}$ (g glucose $\left.)^{-1}\right)$ and productivity $\left(3.1 \mathrm{mmol}\right.$ glycerol $\mathrm{h}^{-1} \mathrm{~g}^{-1}$ dry mass) very similar to those obtained by Geertman et al. (2006) using a different strategy. In the latter case, the authors avoided employing a tpil $\Delta$ mutant while deleting all pyruvate decarboxylase encoding genes in a strain already depleted for the respiratory oxidation of cytosolic NADH. Curiously, this pyruvate decarboxylase negative engineered strain apparently could grow on a glucose mineral medium without the need of acetate, which was in contrast to a previous work by the same group showing that acetate was necessary in the growth medium of a $p d c^{-}$ mutant to provide acetyl-CoA for lipids synthesis (Flikweert et al., 1999). In addition and taking into account our above conclusions, it is very likely that the expression of GPD1 has been increased as a consequence of the engineering strategy followed by these authors (Geertman et al., 2006), since increase of glycerol-3-P dehydrogenase activity is a prerequisite to get a high glycerol flux. Finally, the metabolic engineering for glycerol production incidentally caused several secondary effects that could not be predicted by reconstruction of metabolic networks (Blank et al., 2005; Forster et al., 2003). Further improvement in production and productivity of glycerol should be possible by combining a co-feeding strategy with formate with the continuous removal of glycerol produced. Eliminating interfering $\mathrm{NAD}^{+}$regenerating reactions and expression of a DHAP- insensitive MIP synthase could be also envisaged to evaluate directly the importance of these metabolic reactions in growth efficiency of glycerol engineered strains.

\section{Acknowledgments}

We thank our colleagues for intellectual support during this work and in particular Dr. Thomas Walther for his critical reading of the manuscript. Part of this work was supported by a EU Grant (Project no. QLRT-1999-01364) from the European Commission Framework Programs FP5 to H.C and I.V., and by a one-year Marie Curie young training Fellowships (No. HPMT-EC-2000-00135) to F.M. as part of her Ph.D. Thesis in J.F. laboratory.

\section{Appendix A. Supplementary Materials}

Supplementary data associated with this article can be found in the online version at doi:10.1016/j.ymben. 2007.03.002.

\section{References}

Albertyn, J., van Tonder, A., Prior, B.A., 1992. Purification and characterization of glycerol-3-phosphate dehydrogenase of Saccharomyces cerevisiae. FEBS Lett. 308, 130-132.

Albertyn, J., Hohmann, S., Prior, B.A., 1994a. Characterization of the osmotic-stress response in Saccharomyces cerevisiae: osmotic stress and glucose repression regulate glycerol-3- phosphate dehydrogenase independently. Curr. Genet. 25, 12-18. 
Albertyn, J., Hohmann, S., Thevelein, J.M., Prior, B.A., 1994b. GPD1, which encodes glycerol-3-phosphate dehydrogenase, is essential for growth under osmotic stress in Saccharomyces cerevisiae, and its expression is regulated by the high-osmolarity glycerol response pathway. Mol. Cell Biol. 14, 4135-4144.

Almaguer, C., Mantella, D., Perez, E., Patton-Vogt, J., 2003. Inositol and phosphate regulate GIT1 transcription and glycerophosphoinositol incorporation in Saccharomyces cerevisiae. Eukaryot. Cell 2, 729-736.

Ansell, R., Granath, K., Hohmann, S., Thevelein, J.M., Adler, L., 1997. The two isoenzymes for yeast $\mathrm{NAD}^{+}$-dependent glycerol-3-phosphate dehydrogenase encoded by GPD1 and GPD2 have distinct roles in osmoadaptation and redox regulation. EMBO J. 16, 2179-2187.

Bakker, B.M., Overkamp, K.M., van Maris, A.J., Kotter, P., Luttik, M.A., van Dijken, J.P., Pronk, J.T., 2001. Stoichiometry and compartmentation of NADH metabolism in Saccharomyces cerevisiae. FEMS Microbiol. Rev. 25, 15-37.

Bencini, D.A., Wild, J.R., O’Donovan, G.A., 1983. Linear one-step assay for the determination of orthophosphate. Anal. Biochem. 132, 254-258.

Bisping, B., Rehm, H.J., 1988. Multistep reactions with immobilized microorganisms. Biotechnol. Appl. Biochem. 10, 87-98.

Blank, L.M., Sauer, U., 2004. TCA cycle activity in Saccharomyces cerevisiae is a function of the environmentally determined specific growth and glucose uptake rates. Microbiology 150, 1085-1093.

Blank, L.M., Kuepfer, L., Sauer, U., 2005. Large-scale 13C-flux analysis reveals mechanistic principles of metabolic network robustness to null mutations in yeast. Genome Biol. 6, R49.

Blomberg, A., 1997. Osmoresponsive proteins and functional assessment strategies in Saccharomyces cerevisiae. Electrophoresis 18, 1429-1440.

Blomberg, A., 2000. Metabolic surprises in Saccharomyces cerevisiae during adaptation to saline conditions: questions, some answers and a model. FEMS Microbiol. Lett. 182, 1-8.

Bojunga, N., Entian, K.D., 1999. Cat8p, the activator of gluconeogenic genes in Saccharomyces cerevisiae, regulates carbon source-dependent expression of NADP-dependent cytosolic isocitrate dehydrogenase (Idp2p) and lactate permease (Jen1p). Mol. Gen. Genet. 262, 869-875.

Bun-ya, M., Nishimura, M., Harashima, S., Oshima, Y., 1991. The PHO84 gene of Saccharomyces cerevisiae encodes an inorganic phosphate transporter. Mol. Cell Biol. 11, 3229-3238.

Cambon, B., Monteil, V., Remize, F., Camarasa, C., Dequin, S., 2006. Effects of GPD1 overexpression in Saccharomyces cerevisiae commercial wine yeast strains lacking $A L D 6$ genes. Appl. Environ. Microbiol. 72, 4688-4694.

Campagno, C., Boschi, F., Ranzi, B.M., 1996. Glycerol production in a triose phosphate isomerase deficient mutant of Saccharomyces cerevisiae. Biotechnol. Prog. 12, 591-595.

Casal, M., Paiva, S., Andrade, R.P., Gancedo, C., Leao, C., 1999. The lactate-proton symport of Saccharomyces cerevisiae is encoded by JEN1. J. Bacteriol. 181, 2620-2623.

Chapman, A.G., Atkinson, D.E., 1977. Adenine nucleotide concentrations and turnover rates. Their correlation with biological activity in bacteria and yeast. Adv. Microb. Physiol. 15, 253-306.

Costenoble, R., Valadi, H., Gustafsson, L., Niklasson, C., Johan, F.C., 2000. Microaerobic glycerol formation in Saccharomyces cerevisiae. Yeast 16, 1483-1495.

Cronwright, G.R., Rohwer, J.M., Prior, B.A., 2002. Metabolic control analysis of glycerol synthesis in Sacharomyces cerevisiae. Appl. Environ. Microbiol. 68, 4448-4456.

Drewke, C., Thielen, J., Ciriacy, M., 1990. Ethanol formation in a $a d h^{\circ}$ mutants reveals the existence of a novel acetaldehyde-reducing activity in Saccharomyces cerevisiae. J. Bacteriol. 172, 3909-3917.

Ferreira, C., van Voorst, F., Martins, A., Neves, L., Oliveira, R., Kielland-Brandt, M.C., Lucas, C., Brandt, A., 2005. A member of the sugar transporter family, Stllp is the glycerol/H+ symporter in Saccharomyces cerevisiae. Mol. Biol. Cell 16, 2068-2076.

Flikweert, M.T., de Swaaf, M., van Dijken, J.P., Pronk, J.T., 1999. Growth requirements of pyruvate-decarboxylase-negative Saccharomyces cerevisiae. FEMS Microbiol. Lett. 174, 73-79.
Forster, J., Famili, I., Fu, P., Palsson, B.O., Nielsen, J., 2003. Genomescale reconstruction of the Saccharomyces cerevisiae metabolic network. Genome Res 13, 244-253.

François, J., Van, S.E., Hers, H.G., 1984. The mechanism by which glucose increases fructose 2,6-bisphosphate concentration in Saccharomyces cerevisiae. A cyclic-AMP-dependent activation of phosphofructokinase 2. Eur. J. Biochem. 145, 187-193.

Gancedo, C., Gancedo, J.M., Sols, A., 1968. Glycerol metabolism in yeasts. Pathways of utilization and production. Eur. J. Biochem. 5, $165-172$.

Geertman, J.M., van Maris, A.J., van Dijken, J.P., Pronk, J.T., 2006. Physiological and genetic engineering of cytosolic redox metabolism in Saccharomyces cerevisiae for improved glycerol production. Metab. Eng. 8, 532-542.

Gonzalez, B., Francois, J., Renaud, M., 1997. A rapid and reliable method for metabolite extraction in yeast using boiling buffered ethanol. Yeast $13,1347-1355$

Groussac, E., Ortiz, M., Francois, J., 2000. Improved protocols for quantitative determination of metabolites from biological samples using high performance ionic-exchange chromatography with conductimetric and pulsed amperometric detection. Enzyme Microb. Technol. 26, 715-723.

Guldener, U., Heck, S., Fielder, T., Beinhauer, J., Hegemann, J.H., 1996. A new efficient gene disruption cassette for repeated use in budding yeast. Nucleic Acids Res 24, 2519-2524.

Hochberg, Y., Benjamini, Y., 1990. More powerful procedures for multiple significance testing. Stat. Med. 9, 811-818.

Hohmann, S., 2002. Osmotic adaptation in yeast-control of the yeast osmolyte system. Int. Rev. Cytol. 215, 149-187.

Izawa, S., Sato, M., Yokoigawa, K., Inoue, Y., 2004. Intracellular glycerol influences resistance to freeze stress in Saccharomyces cerevisiae: analysis of a quadruple mutant in glycerol dehydrogenase genes and glycerol-enriched cells. Appl. Microbiol. Biotechnol. 66, 108-114.

Karlgren, S., Pettersson, N., Nordlander, B., Mathai, J.C., Brodsky, J.L., Zeidel, M.L., Bill, R.M., Hohmann, S., 2005. Conditional osmotic stress in yeast: a system to study transport through aquaglyceroporins and osmostress signaling. J. Biol. Chem. 280, 7186-7193.

Klingenberg, M., 1974. Nicotinamide-adenine dinucleotides (NAD, NADP, NADH; NADPH): spectrophometric and fluorimetric methods. In: Bergmeyer, H.U (Ed.), Methods of Enzymatic analysis, vol. 4, pp. $2045-2059$.

Larsson, K., Ansell, R., Eriksson, P., Adler, L., 1993. A gene encoding snglycerol 3-phosphate dehydrogenase $\left(\mathrm{NAD}^{+}\right)$complements an osmosensitive mutant of Saccharomyces cerevisiae. Mol. Microbiol. 10, $1101-1111$.

LeBerre, V., Trevisiol, E., Dagkessamanskaia, A., Sokol, S., Caminade, A.M., Majoral, J.P., Meunier, B., Francois, J., 2003. Dendrimeric coating of glass slides for sensitive DNA microarrays analysis. Nucleic Acids Res. 31, e88.

Louvet, O., Doignon, F., Crouzet, M., 1997. Stable DNA-binding yeast vector allowing high-bait expression for use in the two-hybrid system. Biotechniques 23 (816-8), 820.

Luyten, K., Albertyn, J., Skibbe, W.F., Prior, B.A., Ramos, J., Thevelein, J.M., Hohmann, S., 1995. Fps1, a yeast member of the MIP family of channel proteins, is a facilitator for glycerol uptake and efflux and is inactive under osmotic stress. EMBO J. 14, 1360-1371.

Mewes, H.W., Albermann, K., Heumann, K., Liebl, S., Pfeiffer, F., 1997. MIPS: a database for protein sequences, homology data and yeast genome information. Nucleic Acids Res. 25, 28-30.

Michnick, S., Roustan, J.L., Remize, F., Barre, P., Dequin, S., 1997. Modulation of glycerol and ethanol yields during alcoholic fermentation in Saccharomyces cerevisiae strains overexpressed or disrupted for GPD1 encoding glycerol 3-phosphate dehydrogenase. Yeast 13, 783-793.

Molin, M., Norbeck, J., Blomberg, A., 2003. Dihydroxyacetone kinases in Saccharomyces cerevisiae are involved in detoxification of dihydroxyacetone. J. Biol. Chem. 278, 1415-1423. 
Navarro-Avino, J.P., Prasad, R., Miralles, V.J., Benito, R.M., Serrano, R., 1999. A proposal for nomenclature of aldehyde dehydrogenases in Saccharomyces cerevisiae and characterization of the stress-inducible $A L D 2$ and $A L D 3$ genes. Yeast 15, 829-842.

Nevoigt, E., Stahl, U., 1996. Reduced pyruvate decarboxylase and increased glycerol-3-phosphate dehydrogenase $\left[\mathrm{NAD}^{+}\right]$levels enhance glycerol production in Saccharomyces cerevisiae. Yeast 12, 1331-1337.

Overkamp, K.M., Bakker, B.M., Kotter, P., Luttik, M.A., van Dijken, J.P., Pronk, J.T., 2002. Metabolic engineering of glycerol production in Saccharomyces cerevisiae. Appl. Environ. Microbiol. 68, 2814-2821.

Pahlman, A.K., Granath, K., Ansell, R., Hohmann, S., Adler, L., 2001. The yeast glycerol-3-phosphatases Gpplp and Gpp2p are required for glycerol biosynthesis and differentially involved in the cellular responses to osmotic, anaerobic, and oxidative stress. J Biol. Chem. 276, 3555-3563.

Petrovska, B., Winkelhausen, E., Kuzmanova, S., 1999. Glycerol production by yeasts under osmotic and sulfite stress. Can. J. Microbiol. 45, 695-699.

Petterson, H., Fillipson, C., Becit, E., Brive, L., Hohmann, S., 2005. Aquaporins in yeasts and filamentous fungi. Biol. Cell. 97, 487-500.

Postma, E., Scheffers, W.A., van Dijken, J.P., 1989. Kinetics of growth and glucose transport in glucose-limited chemostat cultures of Saccharomyces cerevisiae CBS 8066. Yeast 5, 159-165.

Remize, F., Roustan, J.L., Sablayrolles, J.M., Barre, P., Dequin, S., 1999. Glycerol overproduction by engineered Saccharomyces cerevisiae wine yeast strains leads to substantial changes in by-product formation and to a stimulation of fermentation rate in stationary phase. Appl. Environ. Microbiol. 65, 143-149.

Remize, F., Barnavon, L., equin, S., 2001. Glycerol export and glycerol-3phosphate dehydrogenase, but not glycerol-3-phosphate phosphatase, are rate limiting for glycerol production in Saccharomyces cerevisiae. Metab. Eng. 3, 301-312.

Remize, F., Cambon, B., Barnavon, L., Dequin, S., 2003. Glycerol formation during wine fermentation is mainly linked to Gpdlp and is only partially controlled by the HOG pathway. Yeast 20, 1243-1253.

Rose, M.D., Winston, F., Hieter, P., 1990. Methods in Yeast Genetics : a Laboratory Course Manual. Cold Spring Harbor Laboratory Press, New York.

Ruepp, A., Zollner, A., Maier, D., Albermann, K., Hani, J., Mokrejs, M., Tetko, I., Güldener, U., Mannhaupt, G., Münsterkötter, M., et al., 2004. The FunCat, a functional annotation scheme for systematic classification of proteins from whole genomes. Nucleic Acids Res. 32, 5539-5545.

Shi, Y., Vaden, D.L., Ju, S., Ding, D., Geiger, J.H., Greenberg, M.L., 2005. Genetic perturbation of glycolysis results in inhibition of de novo inositol biosynthesis. J. Biol. Chem. 280, 41805-41810.
Siderius, M., Van Wuytswinkel, O., Reijenga, K.A., Kelders, M., Mager, W.H., 2000. The control of intracellular glycerol in Saccharomyces cerevisiae influences osmotic stress response and resistance to increased temperature. Mol. Microbiol. 36, 1381-1390.

Sikorski, R.S., Hieter, P., 1989. A system of shuttle vectors and yeast host strains designed for efficient manipulation of DNA in Saccharomyces cerevisiae. Genetics 122, 19-27.

Tamas, M.J., Luyten, K., Sutherland, F.C., Hernandez, A., Albertyn, J., Valadi, H., Li, H., Prior, B.A., Kilian, S.G., Ramos, J., Gustafsson, L., Thevelein, J.M., Hohmann, S., 1999. Fps1p controls the accumulation and release of the compatible solute glycerol in yeast osmoregulation. Mol. Microbiol. 31, 1087-1104.

Teixeira, M.C., Monteiro, P., Jain, P., Tenreiro, S., Fernandes, A.R., Mira, N.P., Alenquer, M., Freitas, A.T., Oliveira, A.L., Sa-Correia, I., 2006. The YEASTRACT database: a tool for the analysis of transcription regulatory associations in Saccharomyces cerevisiae. Nucleic Acids Res. 34, D446-D451.

ter Kuile, B.H., Westerhoff, H.V., 2001. Transcriptome meets metabolome: hierarchical and metabolic regulation of the glycolytic pathway. FEBS Lett 500, 169-171.

Valadi, A., Granath, K., Gustafsson, L., Adler, L., 2004. Distinct intracellular localization of Gpd1p and Gpd2p, the two yeast isoforms of $\mathrm{NAD}^{+}$-dependent glycerol-3-phosphate dehydrogenase, explains their different contributions to redox-driven glycerol production. J. Biol. Chem. 279, 39677-39685.

van Dijken, J.P., Bauer, J., Brambilla, L., Duboc, P., Francois, J.M., Gancedo, C., Giuseppin, M.L., Heijnen, J.J., Hoare, M., Lange, H.C., Madden, E.A., Niederberger, P., Nielsen, J., Parrou, J.L., Petit, T., Porro, D., Reuss, M., van Riel, N., Rizzi, M., Steensma, H.Y., Verrips, C.T., Vindelov, J., Pronk, J.T., 2000. An interlaboratory comparison of physiological and genetic properties of four Saccharomyces cerevisiae strains. Enzyme Microb. Technol. 26, 706-714.

Vries, R.P., Flitter, S.J., van de Vondervoort, P.J., Chaveroche, M.K., Fontaine, T., Fillinger, S., Ruijter, G.J., d'Enfert, C., Visser, J., 2003. Glycerol dehydrogenase, encoded by gldB is essential for osmotolerance in Aspergillus nidulans. Mol. Microbiol. 49, 131-141.

Wach, A., Brachat, A., Pohlmann, R., Philippsen, P., 1994. New heterologous modules for classical or PCR-based gene disruptions in Saccharomyces cerevisiae. Yeast 10, 1793-1808.

White, W.H., Skatrud, P.L., Xue, Z., Toyn, J.H., 2003. Specialization of function among aldehyde dehydrogenases: the $A L D 2$ and $A L D 3$ genes are required for beta-alanine biosynthesis in Saccharomyces cerevisiae. Genetics 163, 69-77.

Woods, R.A., Gietz, R.D., 2001. High-efficiency transformation of plasmid DNA into yeast. Methods Mol. Biol. 177, 85-97. 